Historic, Archive Document

Do not assume content reflects current scientific knowledge, policies, or practices. 



\section{OHIO'S PRIVATE}

\section{OUTDOOR}

A2?5

M2?

AND

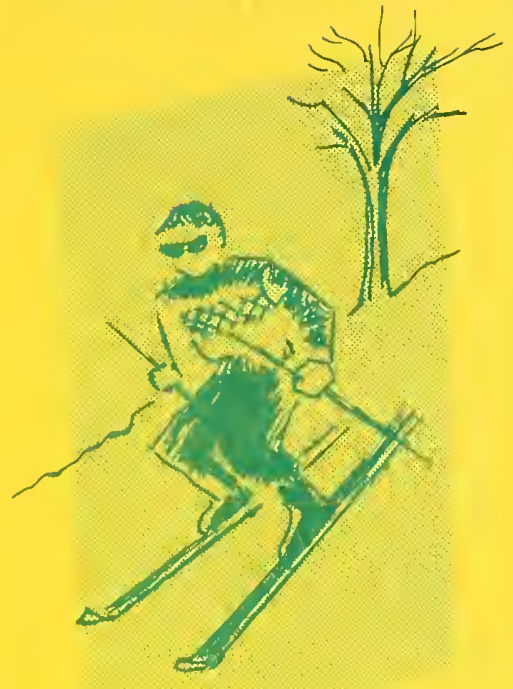

\section{FOREST RECREATION ENTERPRISES}

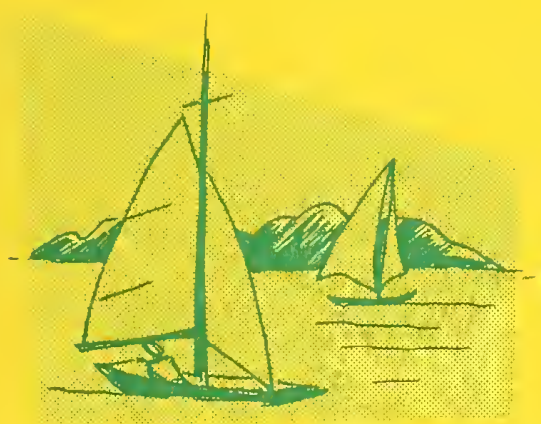

\section{Dwight R. McCurdy}

U. S. OEPT. OF AGRICULTURE

MATIONAL. AGRICUITIJPAL LIORARY

AUG 141963

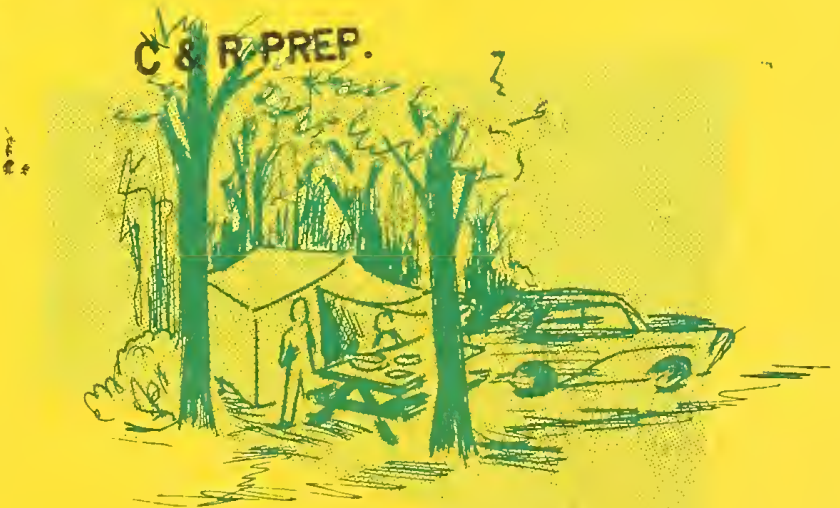

Central States Forest Experiment Station

U.S. Department of Agriculture - Forest Service in cooperation with Ohio Soil and Water Conservation Districts 
Mention of specific enterprises in this

report does not mean endorsement by the publishing agencies.

Omission of any existing enterprises from

the list is an oversight.

\subsection{Exoby?tes}

\section{NATIONAL}

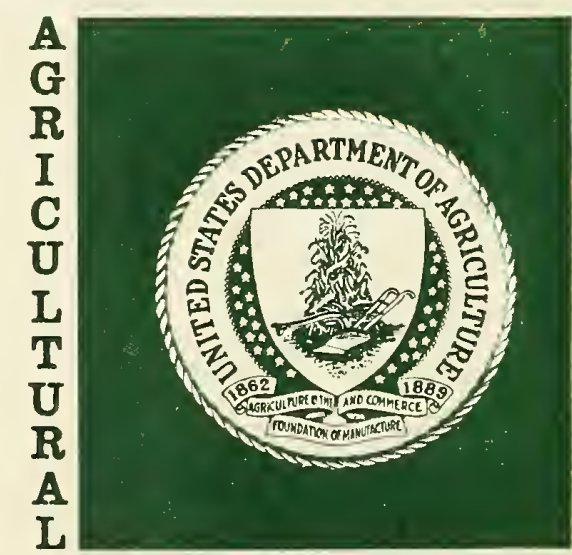

LIBRARY

26655 A225

MP? 


\section{FOREWORD}

The purpose of this directory is to make it easier to gather economic data and other pertinent information for decision making in recreational development. Business, agricultural, and government leaders will be called upon quite frequently in the next few years for this type of information.

The facts presented in this directory were compiled in a cooperative effort and we wish to thank the personnel of Ohio soil and Water Conservation Districts for supplying information on recreational enterprises in their Districts.

Soil and Water Conservation Districts are legal subdivisions of the State of Ohio. Each is governed by five supervisors elected by the owners and occupiers of the land within the district. Districts are organized in Ohio on a county basis. Each develops and carries out a program for the conservation and development of soil and water resources within the district. Programs are carried out through a cooperative agreement with landowners. The goal of every district is use of land within its capabilities.

The Central States Forest Experiment Station is investigating the profit potential of outdoor-recreation enterprises on small private woodlands. This Forest Service research is a part of the Department of Agriculture's broad Rural Area Development program to strengthen rural America.

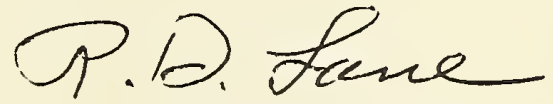

R. D. LANE, Director

Central States Forest Experiment Station

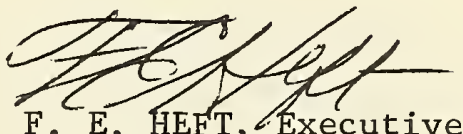

F. E. HEFT, Executive Secretary Ohio Soil and Water Conservation Committee 



\section{OHIO'S PRIVATE OUTDOOR AND}

\section{FOREST RECREATION ENTERPRISES}

\section{Dwight R. McCurdy}

Private enterprises that offer outdoor and forest recreation were inventoried in January and February of 1963 by the Ohio Soil and Water Conservation Districts and the Central States Forest Experiment Station. This directory is the outcome of that inventory.

Names and addresses of those involved in each of the 13 kinds of enterprises are 1isted, beginning on the pages shown below. All charge a fee and, except for some shooting preserves marked with an asterisk $(*)$, all are open to the public.

Page

Campgrounds

Picnic Areas

Cottage Rentals (connected with a farm or forestry enterprise and for fishing or hunting purposes only)

Pay Lakes (fish ponds or lakes -- fishing primarily) 16

Boat Rentals (ponds or lakes -- boating primarily) 24

Private Beaches (ponds or 1akes -- swimming primarily) 26

General Hunting Areas (does not include areas stocked 31 with pen-raised game)

Shooting Preserves (area stocked with pen-raised game) 33

Shooting Areas (includes target and skeet shooting only -- 37 both bows and guns)

Riding Stables

Winter Sports Areas (includes sledding, tobogganing, skiing, etc.)

Organized Summer Camps

Vacation Farms and Dude Ranches 
Personnel of Ohio Soil and Water Conservation Districts revised a list of private recreation enterprises compiled from several sources. District personnel added many unlisted enterprises and made corrections for enterprises that had changed hands or were no longer operating. One source of names and addresses for the preliminary list was case histories of private recreation enterprises in files of the Ohio office of the U.S. Soil Conservation Service. Another U.S. Government source was an inventory of outdoor recreation firms in southeastern Ohio published by Economic Research Service of the Department of Agriculture. The Ohio Department of Natural Resources provided two sources, a list of shooting preserves licensed by Ohio and an inventory of private campgrounds. Two other sources were lists of vacation farms, one published by the Ohio Farm Vacation Association and one published by the Switzerland of Ohio Association.

The following recreational enterprises and organizations were not included in the inventory and do not appear in this directory:

1. Enterprises selling recreational land or leasing recreational rights

2. Associations promoting various recreational enterprises such as the Camping Council, Switzerland of Ohio Vacations Association, etc.

3. Resorts

4. Recreational enterprises adjacent to large bodies of water, such as those on the shores at Buckeye Lake and Lake Erie unless the enterprise was part of a farm

5. Enterprises such as golf courses, driving ranges, dance halls, outdoor theaters, organized sporting areas, go-kart tracks, auto and horse race tracks, swimming pools, etc.

6. Recreational clubs or organizations, such as conservation clubs, country clubs, etc. (except where thought to be open to nonmembers for a fee)

7. Historical sites 


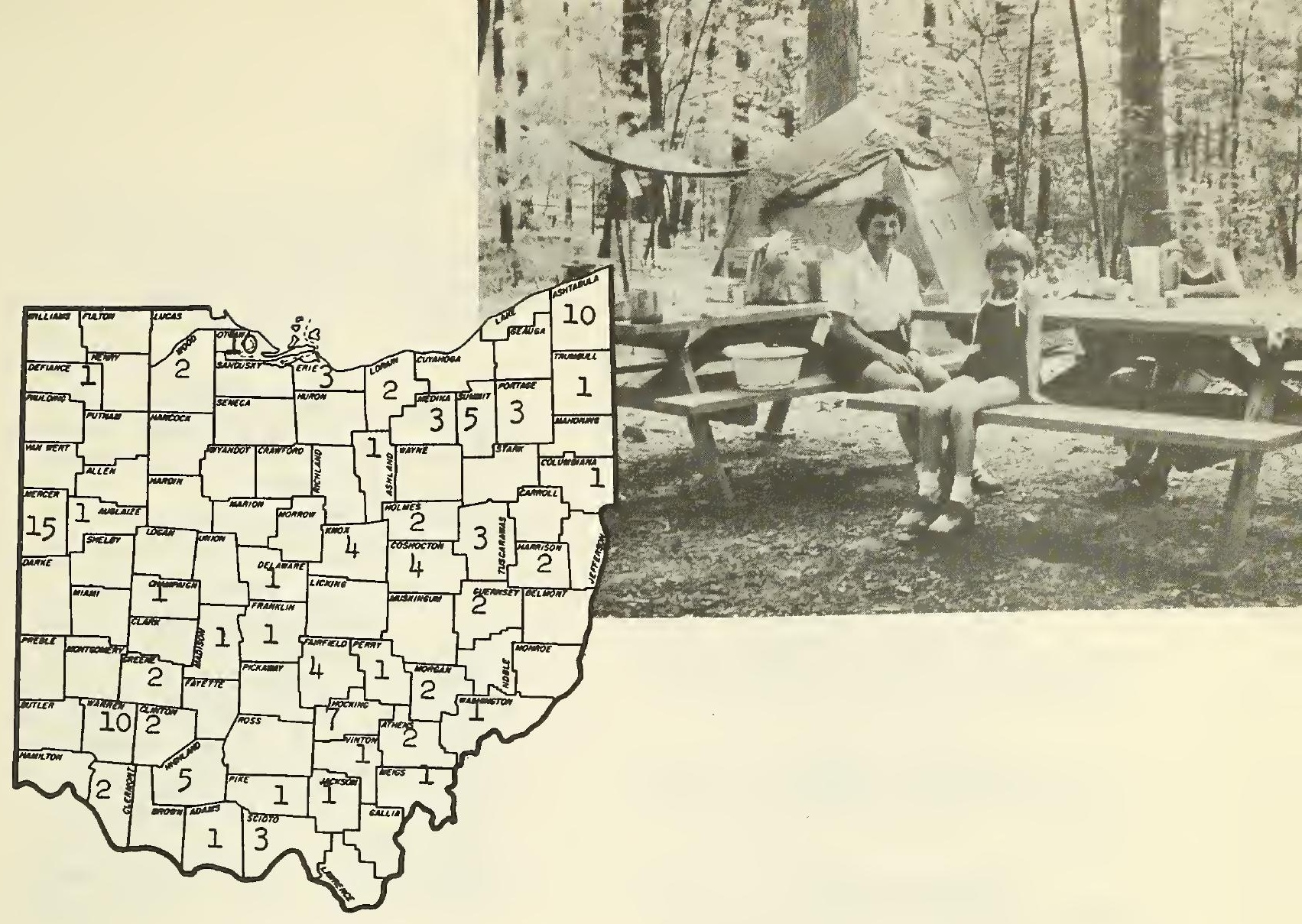

\section{CAMPGROUNDS}

There are generally two types of campgrounds, transient and vacation. The transient campground is located along a well-traveled highway, or close to one, so that travelers may readily stop off while on a trip. It may also be near a large city or some tourist attraction that draws crowds. The vacation campground, on the other hand, is usually a destination in itself and most provide facilities to meet the recreational needs of the camper as well as sleeping and eating needs. Campgrounds are usually found in a forest setting. The fee is generally charged per camping site on a nightly basis.

\section{Adams County}

NORMANDY FRUIT FARM, Car1 R.

Cornvelle, Buena Vista

\section{Ashland County}

NEUBRANDER'S, Art Neubrander, Route 1, Sullivan

\section{Ashtabula County}

BAYSHORE CAMPGROUND, Frank

Caravella, Route 1, Andover
Ashtabula County (cont'd.) CHESTNUT GROVE PART, Geneva DUN ROVIN TRAILER LODGE, Conneaut EVERGREEN LAKE, Conneaut FITTINGS CAMPGROUNDS, Ellen s.

Fitting, Route 1, Lake Road West, Ashtabula LAZY ACRES, R. E. Butler, Route 2, Geneva LOOMAS CAMPGROUND, Ashtabula PIENTERS CAMPGROUND, Ashtabula 
Ashtabula County (cont'd.)

SHADY LANE CAMP, Mr. Ignaut,

Route 1, Andover

WADDLE'S EVERGREEN LAKE, Miller Waddle, 701 Center Road, Conneaut

\section{Athens County}

ANTEL'S CAMPGROUND, Howard Ante1, Bishopville

HANNING'S LAKE, Alton Hanning, Route 5, Athens

\section{Auglaize County}

JONES CAMPING AREA, Dewitt Jones, Route 2, St. Marys

Champaign County

LAKEWOOD BEACH, Herbert

McBride, Route 4, Urbana

\section{Clermont County}

FAY'S CAMPGROUND, Edenton

KRAMERS CAMP, Route 1,

Pleasant Plain

\section{Clinton County}

HUPMAN CAMPGROUND, Pearl Hupman, Route 1, Wilmington

MOORE'S CAMPGROUND, Clarence R. Moore, Route 1, Wilmington

Columbiana County

KREBS CAMPING AREA, John Krebs, Hanoverton

\section{Coshocton County}

COSHOCTON LAKE PARK, Coshocton

OLINGER LAKE, Jack Olinger, Route 1, Coshocton

SUNSET LAKE, Charles Hampton,

Route 1, Coshocton

WILLS CREEK, Coshocton

Defiance County

CAMP LAKOTA, Route 5, Defiance
Delaware County

CAMPLAND, INC., George $\mathrm{H}$. Shapter and Victor Sabo,

Route 2, Delaware

\section{Erie County}

ANDERSON ACRES, INC., Huron

CAMP BOULDER, Raymond Hinde,

Route 1, Huron

MARI-DOR BEACH, Route 1, Vermilion

\section{Fairfield County}

ADENA RIDGE, William Burnside, Route 1, Lancaster

FAIRFIELD COUNTY FISH \& GAME

FARM, Earl R. Ramey, Baltimore

HIDDEN LAKE FARM, Charles

Dunlap, Route 1, Canal

Winchester

JACKSON'S LAKE, S. O. Jackson, Lithopolis

\section{Franklin County}

VALLEY RANCH PICNIC AREA,

Fletcher Audrix, 8802 W.

Broad St., Columbus

\section{Greene County}

HARLAN HURLEY FARM, Harlan Hurley, Route 3, Xenia

SPRING VALLEY TRAILER RANCH,

Mr. Marcus, Spring Valley

\section{Guernsey County}

HOWARD KING FARM, Howard King, Route 1, Cambridge

WILLS CREEK, Coshocton

\section{Harrison County}

HARRISON GUN CLUB, Harold Polen, Uhrichsville

SCIO SPORTSMAN CLUB, Roy Gatts, Scio

\section{Highland County}

BABINGTON CAMP, Emerson Babington, Blanchester 
Highland County (cont'd.) BEECHWOOD, Virginia Layman, 136 W. Main St., Hillsboro BRIGHT ANGEL, Glenn Witters, Route 1, Hillsboro FOREST HILLS, Forest Hill, Route 1, Hillsboro HENNY'S CAMP, Ada Henry, Route 4, Hillsboro

Hocking County CAMP BEUNA VISTA, Charles \& Lawrence Hines, Route 1, Rockbridge

CAMP GREEN HILL, Route 3, Logan CAMP OTTERBEIN, Route 3, Logan COLUMBUS BEAGLE CLUB, Route 3, Logan

FAIRFIELD COUNTY GIRL SCOUT COUNCIL, Mrs. Ralph Heft, Route 2, Logan

LOGAN GIRL SCOUTS COUNCIL, INC. Mrs. Arthur Prey, Route 3, Logan

MATHIAS PARK, Harry Mathias, Route 1, Rockbridge

\section{Holmes County}

PINE VALLEY LAKE, Star Route, Millersburg

PINE VIEW CLUBHOUSE, Orus Weaver, Box 141, Berlin

Jacksom County

CAMP ARROWHEAD, Frank Longstretch and Brooks Jones, Box 388, Jackson

\section{Knox County}

CAMP ANDERSON, John Heinlein, Route 5, Mt. Vernon

CAMP SYCHAR, Melvin Bowers, 201 Sychar Road, Mt. Vernon

CAMP WABENUKA, Richard Boreland, Gambier

THE CAVES, "Bus" Busenburg, Millwood
Lorain County

BERTA'S CAMPING GROUND, Mrs. Berta, Vermilion

HILL BILLY PARK, Joseph Downey, Vermilion

Madison County
DON'S CAMPGROUND, Route 4,
London

Medina County

HILL N' DALE, INC., Gene Tyrre11, Medina

OSAGE LAKE PARK, Roy Lutz, Wad sworth

R-FARM LAKE CLUB, INC., Lodi

MAPLEWOOD LAKE, Gene Hupp, Route 1, Racine

Mercer County
ANDERSON'S LANDING CAMPGROUND,
Boyd Anderson, Route 2,
St. Marys
BASS LANDING CAMPGROUND, Andrew
Bartlett, Route 2, Celina
BAYVIEW, John Van Dyke, Route 2,
Celina
BEHM'S LANDING CAMPGROUND, Mel Miers, Route 2, St. Marys

DOSS LANDING CAMPGROUND, Kenneth Doss, Route 2, Celina

EDGEWATER PARK, David Myers, Route 29 W., Celina

ED'S LANDING CAMPGROUND, Ed Dorsten, Route 2, Celina

FAIRVIEW PARK, Louis Tangeman, Route 29, West Celina

IT'S IT LANDING CAMPGROUND, Ben Huber, Route 2, Celina

SHERMAN'S LANDING CAMPGROUND, William Sherman, Route 2, Celina

SKEEN'S LANDING CAMPGROUND, Stanley Skeen, Route 29, E. Celina

THE MAPLES, David Deeter, Route 2, Celina 
Mercer County (cont'd.)

THE OAKS, D. Chapman, Route 2, Celina

WALKER'S, L. Walker, Route 2,

Celina

WINDY POINT, V. Young, Route 2, Celina

Morgan County

ANTEL'S CAMPGROUND, Howard Antel, Glouster

CAMP SEVENTEEN, Forest

Parmiter, McConnelsville

Ottawa County

CATAWBA ISLAND, Andy Drusbacky

Catawba Island

DOMROSE CAMPGROUND, George

Domrose, Port Clinton

EBERLY CAMPING, Allen Eberly, Route 1, Port Clinton

JIM'S PLACE, L. Weier, Route 2, Port Clinton

LAKESIDE CAMPGROUND, Port

Clinton

L \& N CAMPING, Laurel Neilson, Route 2, Port Clinton

PUT-IN-BAY, Max Weishke,

Put-in-Bay

SHADY SHORES, Richard

Schmardebeck, Route 2, Port

Clinton

WAHLERS FRUIT FARM, L. J.

Wahlers, Route 2, Port

Clinton

ZELLER'S BEACH, Mr. Walker,

Route 2, Port Clinton

Perry County

MASTERSON'S, Roy A. Masterson, Route 2, Crooksville

Pike County

CAVE PARK, Noble White, Washington Court House

Portage County

FRIENDSHIP ACRES PARK, Randolph
Portage County (cont'd.)

HICKORY HILL PARK, Wallace

Girton, New Milford

ROUND-UP LAKE PARK, Mr. Ballonoff, Mantua

CAMP OYO, $\frac{\text { Scioto County }}{\text { Harry Wagner, }}$

112 Chillicothe St., Portsmouth

LAKE MARGARET, Mrs. Margaret

Campbe11, Lucasville

LEWIS LAKE, Otto Lewis, Route 3, Wheelersburg

\section{Summit County}

CRYSTAL LAKE PARK, O. Clare Conlan, Crystal Lake Road, Akron

HILLSIDE PARK, C1inton

LAZY LAKE, Michael Korba, 140 Virginia Kenda11 Road, Peninsula

TAMSIN PARK, Route 1 , Peninsula WYOGO LAKE PARK, James A. Loftus, Jr., 4419 Wyoga Road, Stow

CAMP GREENACRES, Frank

Cunningham, Route 1, Farmdale

Tuscarawas County

DEVILS DEN PARK, N. Storad, Route 2, Tippecanoe

PARADISE LAKES, H. Horsefal1, Route 3, New Philadelphia

TALL TIMBER LAKE, J. Turkovich, Route 1, New Philadelphia

COLUMBUS COMMUNITY COUNCIL, Thelma Stinson, New Plymouth

CAMP BUTTERWORTH, Route 1 , Maineville

CAMP FIREFLY, Route 1, Oregonia

CAMP GRAHAM, Clarksville 
Warren County (cont'd.)

CAMP HOOK, Route 1, Oregonia

CAMP JOY, Rev. Morris McCrackin,

Route 1, Clarksville

CAMP STONYBROOK, Waynesville

CAMP SWANEKY, Route 1, Oregonia

CAMP WA-KA-KEE, Route 1 ,

Oregonia

CAMP WHIP-OR-WILL, Route 1 ,

Morrow

CHURCH OF GOD YOUTH CAMP,

Rev. Steenbargen, Route 1,

Oregonia

Washington County

CHASE' $S$ BEACH, Donn R. Chase,

Little Hocking

Wood County

KNEPPER CAMPGROUND, Wayne

Knepper, Grand Rapids

WINTERGARDEN PARK, Wintergarden,

Road, Bowling Green 


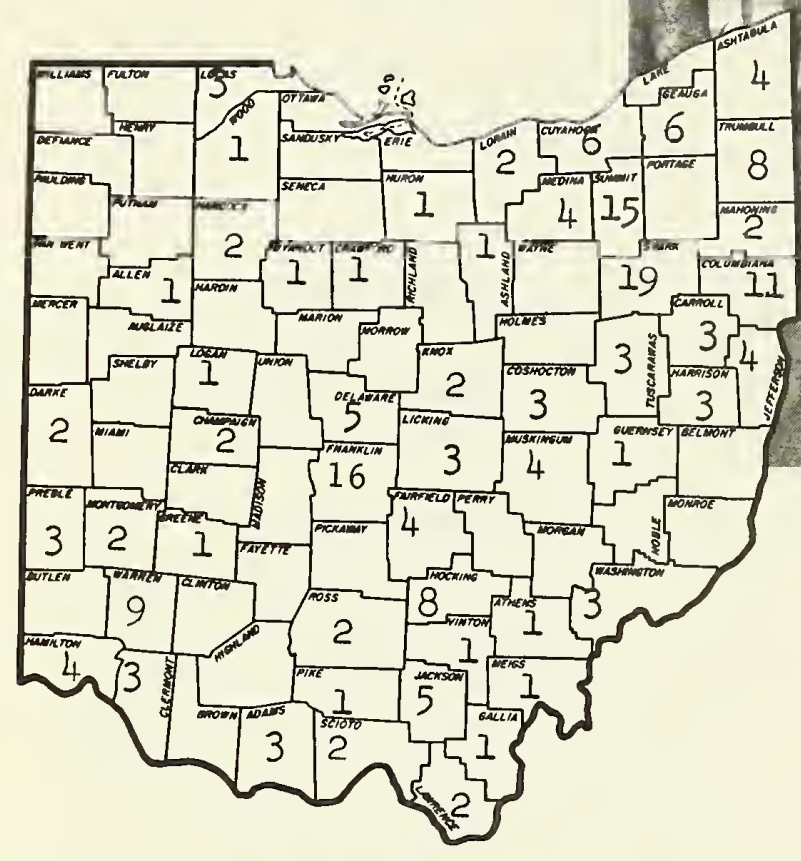

\section{PICNIC AREAS}

Picnic areas can provide several related recreational activities. They are often used by groups for a few hours or all day, and center around water and trees. A general admission fee is usually charged with additional fees for participation in particular activities. Facilities range from one picnic table under a shade tree or a group of tables in a woodlot to large open areas suitable for outings of carnival aspect. Picnicking encourages participation in other outdoor activities. Therefore, areas and facilities suitable for games, hiking, swimming, walking for pleasure, etc. are usually provided.

Adams County

ADAMS COUNTY BEAGLE CLUB,

T. W. Eylar, Winchester

TUCKER'S POND, Harry Tucker,

Route 2, Manchester

WREN'S PARK, C. D. Wren,

West Union

\section{Allen County}

TWIN ROCK LODGE, J. E. Theising, Route 5, Lima
Ashland County

NEUBRANDER'S, Art Neubrander, Route 1, Sullivan

Ashtabula County

BUCK LAKE, Conneaut

LAZY ACRES, R. E. Butler, Route 2, Geneva

LIMBACH PICNIC AREA, P1ymouth Township

WADDLE'S EVERGREEN LAKE, Miller Waddle, 701 Center Road, Conneaut 
Athens County

HANNING'S LAKE, A1ton Hanning, Route 5, Athens

\section{Carrol1 County}

BILLY B. BEACH, Ruth Blackstone, Route 1, Carrol1ton

FOLTZ LAKE, James Foltz, Malvern VO-ASH LAKE, Steve Neggett, Route 3, Carrollton

\section{Champaign County}

LAKEWOOD BEACH, Herbert McBride, Route 4, Urbana

OHIO CAVERNS, Marion Smith, Route 1, West Liberty

Clermont County

CEDAR FISHING LAKE, Smith Road, Route 3, Lovel and

LAKE CAST A LINE, Route 2, Lovel and

MUELLER'S LAKE CLUB, Paul Mueller, Route 2, Loveland

\section{Columbiana County}

DILL LAKE, Fred Dill, Columbiana

CALDWELL LAKE, James Caldwell, Route 1, Rogers

COPELAND LAKE, W. L. Vaughn, Leetonia

JENKINS LAKE, Sterling Jenkins, Route 1, Salineville

LAKE CHA-VEL, David Tice, Route 1, Wellsville

LAKE ELDORADO, John Bogdon, Route 4, Lisbon

LITTLE ROCK, Charles Bibbee, Route 1, East Liverpool

MAR-VIN LAKE, F. D. Irwin, Radio Heights, East Liverpool

SALEM RESERVOIR, Ralph Harker, Route 2, Salem

TERRYS LAKE CORP., Willard Stol1, Route 2, Lisbon

WESTVILLE LAKE, Allen Craven, Westville
Coshocton County

BARTH LAKE, Fred Barth, Route 2, Fresno

OLINGER LAKE, Jack Olinger, Route 1, Coshocton

SUNSET LAKE, Charles Hampton, Route 1, Coshocton

\section{Crawford County \\ SUNSET SPRINGS, George Preston, Osceola}

BROADVIEW LAKE PARK, 8628

Broadview Road, Clevel and

DRANEK'S ASTORHURST, 6980 Dunham, Bedford

FORESTVUE PARK, 9377 Ridgewood Drive, Clevel and 24

SHERWOOD FOREST, 12820 Tinkers Creek Road, Cleveland 25

SIEDEL LAKE, Norbert Siedel, 21897 Westwood Drive, Strongsville 36

WEST SALLY GROVE, Sally West, W. 130th-1/2 Mile South of Albion, Strongsville 36

\section{Darke County}

STILLWATER BEACH, Route 1, Brad ford

WAYNE LAKES, Lowe11' Vietor, Route 1, Greenville

\section{Delaware County}

HOLIDAY HILL, John Rowlands, Route 3, Delaware

MACKS PARTY HOUSE, Don Mack, Route 2, Delaware

RATHBONE RANCH, Howdy Gorman, Route 3, Delaware

WRFD PICNIC AREA, Worthington YARNELL'S PARTY HOUSE, Carrol1

Yarne11, Route 2, Westerville

Fairfield County

ADENA RIDGE, William Burnside, Route 1, Lancaster 


\section{PICNIC AREAS (cont'd.)}

Fairfield County (cont'd.) HICKORY LAKE, Roman DeGuzman, Route 1, Lancaster

HIDDEN LAKE FARM, Charles Dunlap, Route 1, Canal Winchester SPRING LAKES, R. T. Whitehead, Refugee Road, Pickerington

Frank lin County

AUNT NETTIES HIDEWAY, Cleveland Ave. (north of 161), Columbus

CUBBAGE CORNERS, 4296 E. Walnut, Columbus

ECHO WOODS PARK, 9171 E. Main St., Columbus

EDGEWATER PARK, 4400 Floyd Drive, Columbus

FLINT PARK, 225 Park, Columbus GOODING ZOO AMUSEMENT PARK, 8888 Riverside Drive, Columbus

HEMPY'S GROVE, Refugee Road, Columbus

LIVING WATERS PRIVATE PICNIC GROUNDS, Powe11 Road, Columbus

MURPHY 'S PARTY BARN \& PICNIC GROUNDS, State Route 750, Powe11

NEDELMAN RIDING STABLES, Nathan Nedelman, Waggoner Road, Columbus

OAK PARK, 5442 Sunbury Road, Columbus

SMITH LAKE, Cecil Smith, Lambert Road, Columbus SOUTHWESTERN FISHING CLUB, William H. C1ick, 1571 Demorest, Columbus

SPOTTSWOOD VIOLA BOOKER MEMORIAI PARK, Groveport Road, Columbus

VALLEY RANCH PICNIC AREA, Fletcher Audrix, 8802 W. Broad St., Columbus
Franklin County (cont'd.) WOLLERMAN'S RECREATION CENTER, Vernon Wollerman, Bixby Road, Columbus

\section{Gallia County}

CORA PARK, Mrs. Paul Jones, Route 2, Gallipolis

Geauga County

BRIDGE ROAD LAKES, Warren Rondini, Middlefield

BROKEN BIT RANCH, Blanche Fleder, Garrettsville

HOSMER LAKES, Frank and Robert

Hosmer, Burton

OCEANA PARK, M. Matrai, Route 1, Burton

ROSE FARM, Myron Rose, Russe11 SPRING LAKE PARK, Manny Fendrich, East Claridon

\section{Greene County}

HARLAN HURLEY FARM, Harlan

Hurley, Route 3, Xenia

\section{Guernsey County}

HOWARD KING FARM, Howard King, Route 1, Cambridge

\section{Hamilton County}

BALDY'S FISHING LAKES, 5136 Foley, Cincinnati 38

HAMILTON COUNTY PARK, 1000 Miles Road, Cincinnati 31

KRAMERS FISHING LAKE, 3340 West Galbraith, Cincinnati

LAKE NINA, 7200 Pippin Road, Cincinnati

Hancock County

BENROTH RECREATION CLUB, Z. M. Benroth, Route 5, Findlay BLUE PIGEON, Cletis Winstead, Route 5, Findlay 
Harrison County

CADIZ LIONS CLUB, Austin Wyckoff, Cadiz

HARRISON GUN CLUB, Harold

Polen, Uhrichsville

SCIO SPORTSMAN CLUB, Roy Gatts, Scio

\section{Hocking County}

CAMP AKITA, Route 2, Logan

CAMP BEUNA VISTA, Charles and Lawrence Hines, Route 1, Rockbridge

CAMP GREEN HILL, Route 3, Logan CAMP OTTERBEIN, Route 3, Logan COLUMBUS BEAGLE CLUB, Route 3, Logan

FAIRFIELD COUNTY GIRL SCOUT COUNCIL, Mrs. Ralph Heft, Route 2, Logan

LOGAN GIRL SCOUTS COUNCIL, INC., Mrs. Arthur Prey, Route 3, Logan

MATHIAS PARK, Harry Mathias, Route 1, Rockbridge

\section{Huron County}

LOVERS LANE GOLF COURSE, George and John Gens, Route 3, Norwalk

\section{Jackson County}

BAILEY LAKE, John Bailey, Route 5, Jackson

CANTER'S CAVE, Rodney Marhoover, Box 110, Jackson

RICHARDS PAY LAKE, Cecil Richards, Route 2, Wellston

TRIPPIES LAKE, Chester Triplett, Route 1, Ray

VAN FOSSEN LAKE, Charles Van Fossen, Route 3, Jackson

\section{Jefferson County}

AUSTIN LAKE, INC., Glenn Cable, Route 1, Irondale

FRED'S LAKE, Route 1, Richmond GEORGE'S LAKE, Thomas George, Bergholz
Jefferson County (cont'd.)

MALABU LAKE, Donald and David. Pesta, 1205 Lincoln Ave., Steubenville

\section{Knox County}

CAMP ANDERSON, John Heinlein, Route 5, Mt. Vernon

THE CAVES, "Bus" Busenburg, Millwood

GINA LAKE, Coal Grove

LAWCO LAKE ASSOCIATION, Ironton

Licking County

CHAPMAN'S SUN VALLEY LAKE, Kenneth Chapman, Dayton Road, Route 1, Newark

DAR-LEE SHOOTING RANGE, 388 Taylor Road, Summit Station

LAKE OTTO, Calvin Stock1i, Route 3, Frazeysburg

\section{Logan County}

AL-KEN LAKES, K. J. Roberts, Route 2, Bellefontaine

\section{Lorain County}

RASHES, A. E. Rash, Crownhill St., Amherst

WAGNERS, James Wagner, 1119 Middle Ridge Road, Amherst

BENNARD'S PICNIC AREA, 8052 W. Central, Toledo CENTENNIAL RECREATION CLUB, 1943 Centennial Ave., Toledo OAK SHADE, Andy Eishen, 3624 Seaman Road, Toledo

\section{Mahoning County}

ARROWHEAD LAKE PARK, Fred Di11, Columbiana

WILDWOOD LAKE PARK, Car1 Shank, North Lima 


\section{Medina County}

GRANGER LAKE, Route 2, Medina

HILL N' DALE, INC., Gene Tyrre11, Medina

MACK'S LAZY T, John C. Mack, Valley City

OSAGE LAKE PARK, Roy Lutz, Wadsworth

\section{Meigs County}

MAPLEWOOD LAKE, Gene Hupp, Route 1, Racine

Montgomery County DENLINGER PICNIC AREA, Harry Denlinger, Wolf Creek Pike at Olive Road, Dayton 26 NORTHRIDGE FISHING LAKE, 4599 North Dixie Drive, Dayton 14,

\section{Muskingum County}

DIEHR LAKE, Walter Diehr, East Fultonham

LAKE ISABELLA, East Fultonham LAKE VIEW, Robert Hopper, Pinkerton Lane, Route 2,

Zanesville

NORTHSIDE BEACH, Larry Finan, Frazeysburg Road, Zanesville

CAVE PARK, $\frac{\text { Pike County }}{\text { Noble White }}$ Washington Court House

\section{Preble County}

BERRY LAKES, Eaton Lewisburg Road, Lewisburg

FRANCE PARK, New Paris MARVA LAKES, Dewey Walton, Route 2, New Paris

\section{Ross County}

ATER'S LAKE, Raymond Ater, Route 3, Chillicothe

LAKE HILL, Hill Brothers, Frank fort
Scioto County

LAKE MARGARET, Mrs. Margaret Campbell, Lucasville

LEWIS LAKE, Otto Lewis, Route 3, Wheelersburg

ATWATER PARK, Magadore Road, Uniontown

CLEMENS GROVE, Andrew Clemens, 2707 Klotz Ave., East Canton CRYSTAL LAKE PARK, 4848 Crystle Lake Road, Massillon

ECHO VALLEY FARM, Robertsville FOUNTAIN BLUE, 5567 Earl Road, Massillon

HIDDEN VALLEY LAKE, Donald Cole, Baird Road, Paris

HIGH MILL PARK, Mrs. Sanders, High Mill Ave., Massillon LAKE JOSEPH, Gust Winkler, Jr., 1433 Dowing St., East Sparta

LAKE SHERMAN, Lake Drive, Navarre

LOUIES PICNIC GROVE, Hoover N. W., Hartville

LYN NITA PICNIC GROVE, Lynn

Rhinhart, Sherman Church Ave., Canton

MENEGAY'S PARK, Richard Menegay, Louisville

MUZZY LAKE, James Muzzy, 830 - 38th N. W., Canton

PLEASANT LAKE PARK, Pleasant Lake Road, East Sparta

PLEASANT VALLEY LAKE, Tony Stefanick, Route 1, East Canton STARRY HI, INC., O. F. Baires, 2664 Belfort Ave., Louisville SUNSET PARK, Robert Hostetter, Edison St., Alliance

VILLA-PADOVA, 5650 Stuber Drive, Canton

WILLOW SPRINGS, 1715 - 55th St., N. W., Canton

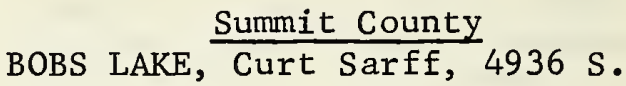
Arlington St., North Canton 20 
Summit County (cont'd.)

CARTER LAKES, Harold Carter, 9032 Charles Ct., Twinsburg

CRYSTAL LAKE PARK, O. Clare Conlan, Crystal Lake Road, Akron

FELL LAKE PARK, Tony Perko, 10070 Akron-Cleveland Road, Northfield,

HILLSIDE PARK, Clinton

HUDSON SPRINGS PARK, Michael C. May, 15 Baldwin, Hudson

KOOL LAKE PARK, George Kuli, 1043 Hardy Road, Cuyahoga Fal1s

LAKE KTM-TAM, C. W. Patton, 194 Praire Drive, Akron 12

LOYAL OAK LAKE PARK, Robert Emery, 2678 S. Hametown Road, Barberton

MACA PARK, Irwin and Don Bowsher, 988 Beechwood Drive, Tallmadge

MUNROE FALLS PARK, Jack Renner, Munroe Falls

SILVER SPRINGS PARK, Russel1

Karas, 5027 Stow Road, Stow

TALLMADGE SPRINGS PARK, INC., Dennis Barnes, 619 Northwest Ave., Tallmadge

TAMSIN PARK, Route 1, Peninsula

WYOGO LAKE PARK, James A. Loftus, Jr., 4419 Wyoga Road, Stow

ANDERSON'S LAKE, Robert G. Anderson, Route 1, Vienna

CAROMA RANCH, Mark Hal1, Route 1, Lockwood

FARMER JIM'S, B. A. Callahan, 1610 Niles-Cortland Road, Cortland

HAWTHORNE LAKE, Don Rodney, Route 1, Bristolville

NOBLE'S PICNIC GROUNDS, NobleGoodhart, Route 1, Cortland SMELKO'S, A1 Smelko, 1955 NilesCortland Road, Cortland $\frac{\text { Trumbul1 County (cont'd.) }}{\text { ING LAKE, Fred Newberry, }}$

Bristolville

WILLOW LAKE PARK CLUB, Nick Dan,

6863 Mahoning N. W., Warren

Tuscarawas County

DEVILS DEN PARK, N. Storad, Route 2, Tippecanoe

PARADISE LAKES, H. Horsefall, Route 3, New Philadelphia

RAINBOW LAKES, Nick Pappas, Route 1, Newcomerstown

COLUMBUS COMMUNITY COUNCIL,

Thelma Stinson, New Plymouth

Warren County

ARMCO ASSOCIATION PARK,

Route 3, Lebanon

CAMP BUTTERWORTH, Route 1 , Maineville

CAMP GRAHAM, Clarksville

CAMP JOY, Rev. Morris McCrackin,

Route 1, Clarksville

CHURCH OF GOD YOUTH CAMP, Rev.

Steenbargen, Route 1, Oregonia

GINE-BELL LAKE, Route 1, Morrow

KETTERING BRANCH - YMCA,

Route 2, Waynesville

PINEY WOODS, A. E. Switzer,

Route 1, Morrow

WUNKER'S LAKE, R. B. Wunker,

Shadow Lake

CIVITAN $\frac{\text { Washington County }}{\text { PARK, Marietta }}$

MASONIC PARK, Marietta

SIEDA, Carl L. Weppler \& Albert

Berg, Route 2, Whipple

Wood County

WINTERGARDEN PARK, BOWling Green

Wyandot county

CAREY SPORTSMEN CLUB, Carey 


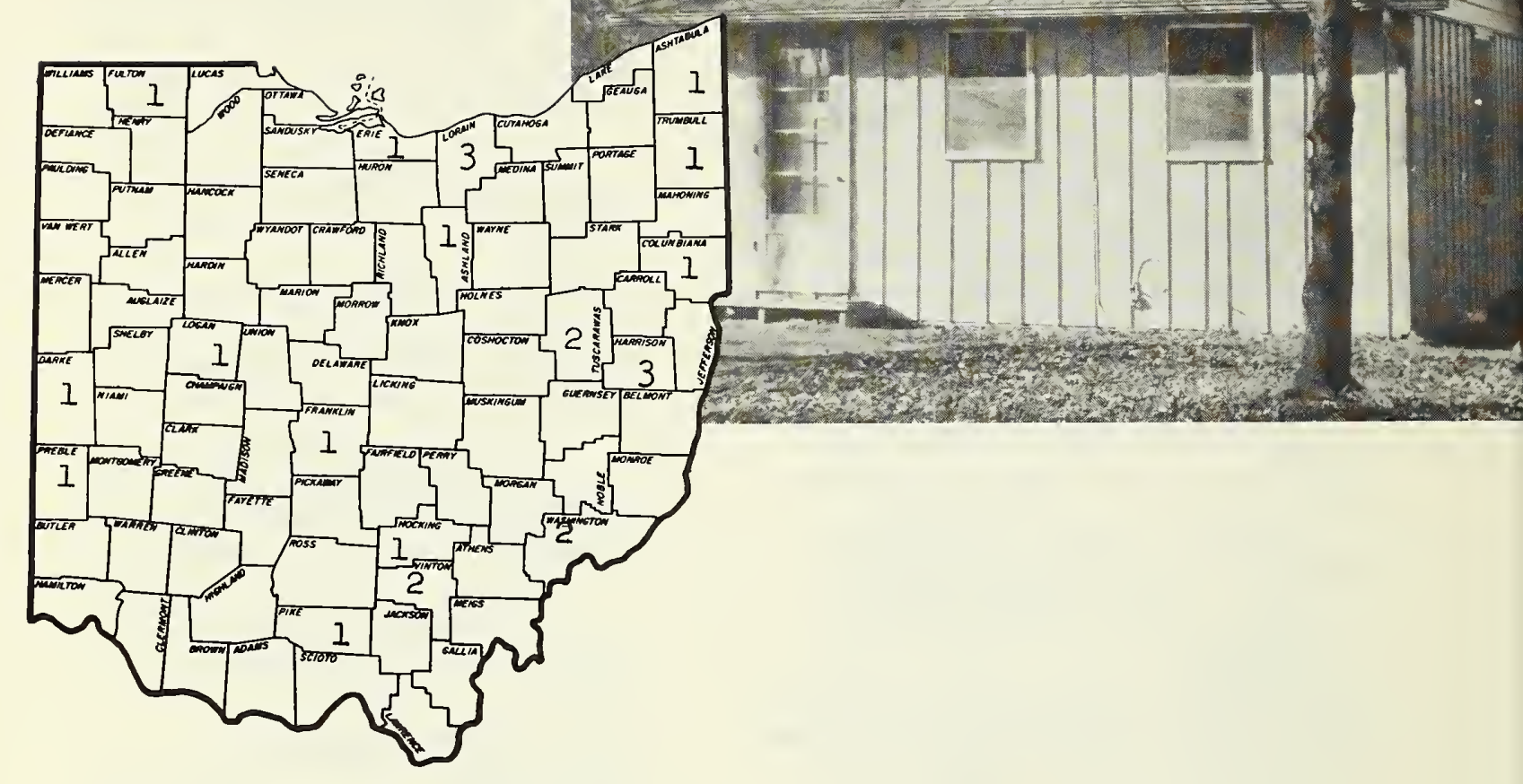

\section{COTTAGE RENTALS}

The inventory included only cottages owned by farmers or forest-1and owners and rented for fishing or hunting. The facilities ranged from rustic cabins located along streams running through the forest, with little or no furnishings, to modern cabins along well-traveled highways, with everything furnished including bedding. The fee is usually on a daily or weekly basis. Many cabins are associated with a campground or pay lake.

\section{Ashland County}

NEUBRANDER'S, Art Neubrander, Route 1, Sullivan

\section{Ashtabula County}

FITTINGS CAMPGROUNDS, El len s. Fitting, Route 1, Lake Road West, Ashtabula

\section{Columbiana County}

LAKE ELDORADO, John Bogdon, Route 4, Lisbon

\author{
Darke County \\ WAYNE LAKES, Lowe11 Vietor, \\ Route 1, Greenville
}

$\frac{\text { Erie County }}{\text { MARI-DOR BEACH, Route 1, Vermilion }}$
$\frac{\text { Frank1in County }}{\text { VALLEY RANCH PICNIC AREA, Fletcher }}$
Audrix, 8802 W. Broad St.,
Columbus




\section{COTTAGE RENTALS (cont'd.)}

Fulton County

L. STEENSON, L. Steenson, Route 1, Fayette

Harrison County

CLENDENING COTTAGE RENTAL, W. B. Bose, Freeport

HAMILTON'S, Albert Hamilton, Route 2, Freeport

TAPPAN COTTAGE RENTAL, Lloyd and Max Holleyoak, Route 1, Scio

Hocking County

CAMP BEUNA VISTA, Charles and

Lawrence Hines, Route 1, Rockbridge

\section{Logan County}

AL-KEN LAKES, K. J. Roberts, Route 2, Bellefontaine

Lorain County

BUCHS PLACE, Harold Buchs, Route 1, Oberlin

DAN CAMPBELL'S FARM COTTAGES, Dan Campbe11, Route 1, Oberlin GEST'S COTTAGES, Herman Gest, Route 2, Grafton

\section{Pike County}

VULGAMORE COTTAGE RENTAL, Leo

Vulgamore, Star Route,

Lucasville

\section{Preble County}

FRANCE PARK, New Paris

Trumbu11 County

CAROMA RANCH, Mark Hal1, Route 1, Lockwood

Tuscarawas County

DEVILS DEN PARK, N. Storad, Route 2, Tippecanoe

TALL TIMBER LAKE, J. Turkovich, Route 1, New Philadelphia

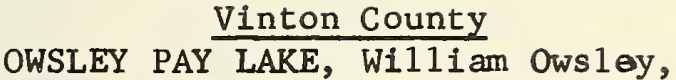
Route 2, McArthur

ZINN PAY LAKE, Ernest Zinn, Hamden

Washington County

LAKESIDE, Chop Ullman, Beverly SECOY LAKE, Car1 Secoy, Barlett 


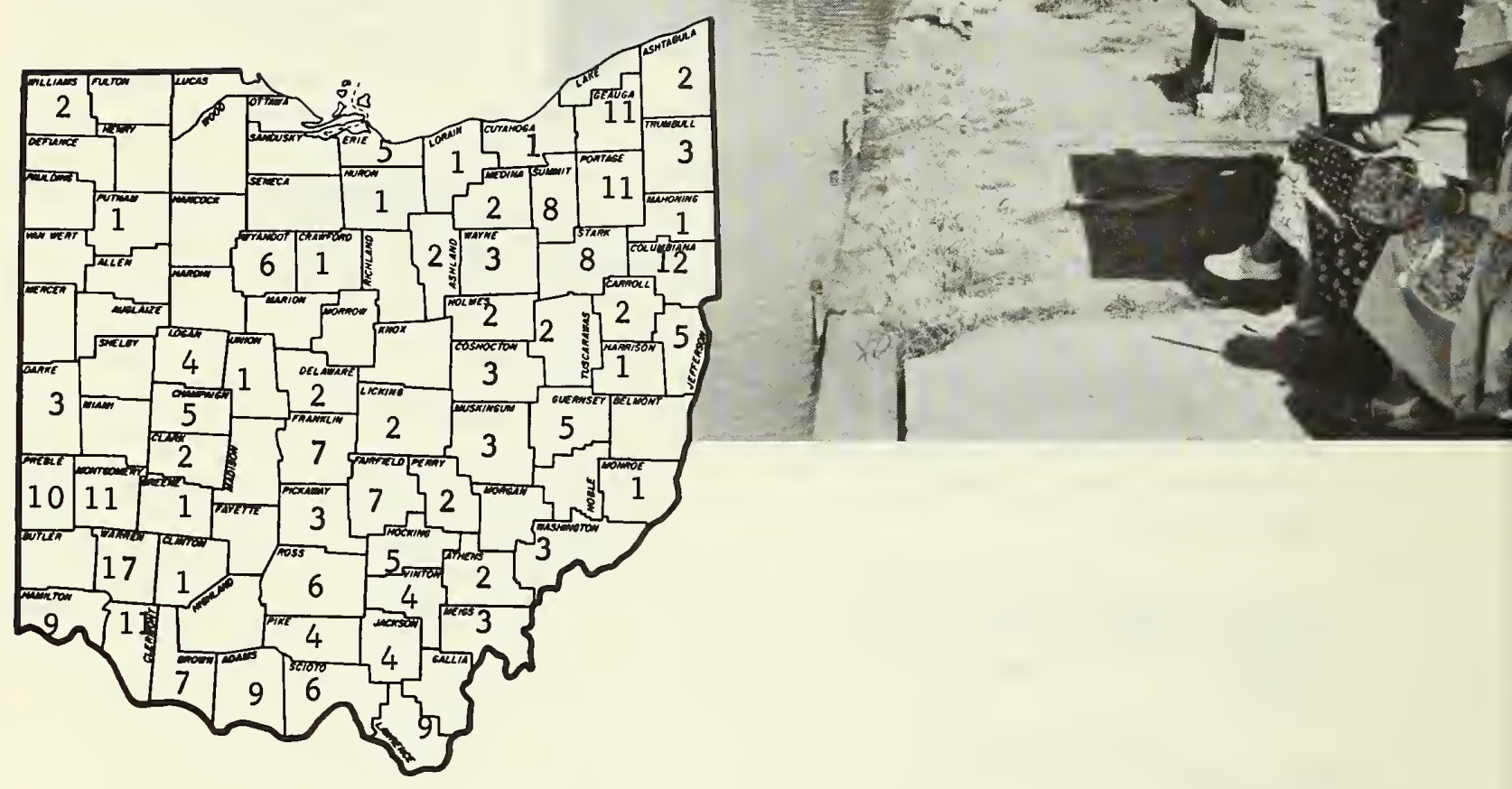

\section{PAY LAKES}

Pay fishing ponds or lakes are often stocked with particular species of fish such as northern pike, walleyes, rainbow trout, etc. Usually, if a variety of fish is offered, there will be several ponds or lakes. Such facilities as boats and boat ramps are often available. Snackbars, picnic areas, sale of tackle and bait, etc. may be provided. The fee is generally charged per pole or on a daily basis. But ponds or lakes are also often leased on an annual basis to an organized group.

DORTON'S LAKE, E. D. Dorton, West Union

DAY'S FISHING LAKE, J. E. Day, Route 1, Winchester

GARRISON'S LAKE, Wayne Garrison, Route 1, Seaman

HIDEAWAY LAKE, William Moore, West Union

MITCHELL'S LAKE, Richard $W$.

Smith, Route 2, Peebles

RIDGE LAKES, Arthur Reece,

Route 3, West Union
Adams County (cont'd.) SHAWS FISHING LAKE, Harold Shaw, Winchester

STONE LAKE, James H. Stone, Route 2, Peebles

TUCKER'S POND, Harry Tucker, Route 2, Manchester

\section{Ash1and County}

METCALF LAKE, H. E. Morr,

Route 2, Lakeville

NEUBRANDER'S, Art Neubrander, Route 1, Sullivan 


\section{Ashtabula County}

BUCK LAKE, Conneaut

LAZY ACRES, R. E. Butler,

Route 2, Geneva

\section{Athens County}

BUCKS LAKES, H. C. Buck, Route 2, Guysville

HANNING'S LAKE, Alton Hanning, Route 5, Athens

\section{Brown County}

ADKINS' LAKE, Orville Adkins,

Route 1, Hamersville

ERNST'S LAKE, Joseph Ernst, 403 Home St., Georgetown

LIMING'S LAKE CLUB, Richey Liming, Route 1, Hamersville

PLEASURE LAKE, Ova Anderson,

Route 2; Mt. Orab

RICKEY'S LAKE, Ed Rickey, Georgetown

SPRING VALLEY LAKE, Clarence

Hunley, Kline Road, Sardinia

WILLOW LAKE, W. A. Colwe11,

Route 1, Bethel

\section{Carrol1 County}

FOLTZ LAKE, James Foltz, Malvern

VO-ASH LAKE, Steve Neggett, Route 3, Carrollton

\section{Champaign County}

BREEDLOVE POND, Dewitt Weller, Route 1, Urbana

LAKEWOOD BEACH, Herbert MCBride, Route 4, Urbana

MOODY LAKE, Edgar Moody, Route 1, Cable

STROMAN LAKE, Charles Stroman, Route 2, St. Paris

VAN DARBY CLUB, Route 2, Mechanicsburg

\section{Clark County}

BEAVER LAKE FISHING CLUB, Edwin Kirby, Mounted Route 52, Springfield
Clark County (cont'd.)

MOE'S LAKE, Sam Grenet, Route 1, South Vienna

\section{Clermont County}

BELL'S LAKES, Clarence Bailey, 575 Marjorie Drive,

Cincinnati 44

BEULAH LAKE, Mr. Maufus, Mt.

Carme1, Cincinnati 44

CEDAR FISHING LAKE, Route 3, Lovel and

DELA PALMA FISHING LAKE, Route 1, Williamsburg

HIDDEN HILL LAKE, Ray Dayton, Route 2, New Richmond

LAKE CAST A LINE, Route 2, Lovel and

LAKE MONTEREY, Route 4, Batavia

LEN'S LAKE CLUB, Len Wadde11, Route 2, Batavia

MUELLER'S LAKE CLUB, Paul Mueller, Route 2, Lovel and

OWENSVILLE FISHING LAKE, Mrs. Harry W. Dodge, Route 3, Batavia

RAPP'S CAMP GROUND, Route 1 , Felicity

\section{Clinton County}

SCHMIDT LAKE, Kar1. Schmidt, Route 3, Wilmington

AL'S LAKE, Albert Raabe

Route 2, Leetonia

CALDWELL LAKE, James Caldwe11, Route 1, Rogers

CONSER RUN, Theodore and Kent Sanor, Route 1, East Rochester COPELAND LAKE, W. L. Vaughn, Leetonia

JENKINS LAKE, Sterling Jenkins, Route 1, Salineville

LAKE CHA-VEL, David Tice, Route 1, Wellsville

LAKE ELDORADO, John Bogdon, Route 4, Lisbon 
Columbiana County (cont'd.)

MAR-VIN LAKE, F. D. Irwin, Radio

Heights, East Liverpool

MCBURNEY'S LAKE, W. H. McBurney, Rogers

SALEM RESERVOIR, Ralph Harker, Route 2, Salem

TERRYS LAKE CORP., Willard

Stol1, Route 2, Lisbon

WESTVILLE LAKE, Allen Craven, Westville

\section{Coshocton County}

BARTH LAKE, Fred Barth, Route 2, Fresno

OLINGER LAKE, Jack Olinger, Route 1, Coshocton

SUNSET LAKE, Charles Hampton, Route 1, Coshocton

\section{Crawford County}

SUNSET SPRINGS, George Preston, Oceola

\section{Cuyahoga County}

SIEDEL LAKE, Norbert Siedel, 21897 Westwood Drive,

Strongsville 36

\section{Darke County}

PAUL'S LAKE, Paul Reeser, Route 1, Bradford

SUGAR VALLEY LAKE, Route 4, Greenville

WAYNE LAKES, Lowe11 Vietor, Route 1, Greenville

Delaware County

POPPLER LAKE, Route 1, Sunbury

RAINBOW LAKE, Center Village

\section{Erie County}

ANDERSON ACRES, INC., Huron

BAY BRIDGE MARINA, Cliff Myers, Bay View

CASTALIA TROUT CLUB, Everett Reeves, Castalia
Erie County (cont'd.)

DEER PARK, Herbert Nielsen, Route 2, Martins Point Road, Sandusky

ROCKWELL SPRINGS TROUT CLUB, Route 1, Vickery Road, Castalia

\section{Fairfield County}

ADENA RIDGE, William Burnside, Route 1, Lancaster

ANGLER'S PARADISE CLUB, Sugar Grove

FAIRFIELD COUNTY FISH \& GAME FARM, Ear1 R. Ramey, Baltimore

HICKORY LAKE, Roman DeGuzman, Route 1, Lancaster

HIDDEN LAKE FARM, Terry and Charles Dunlap, Route 1, Canal Winchester

SPRING LAKES, R. T. Whitehead, Refugee Road, Pickerington

TRIANGLE LAKE, Paul E. Schweikert, Route 3, Lancaster

BLACKLICK RIDING \& FISHING CLUB, 1253 Waggoner, Columbus

IVY WOOD LAKES, Perrill Road, Columbus

JACKSON LAKE, Cedar Hill Road, Columbus

NEDELMAN LAKE, Nathan Nedelman, Waggoner Road, Columbus

SMITH LAKE, Cecil Smith, Lambert Road, Columbus

SOUTHWESTERN FISHING CLUB,

William H. Click, 1571 Demorest, Columbus

VALLEY RANCH PICNIC AREA, Fletcher Audrix, 8802 W. Broad St., Columbus

\section{Geauga County}

BLUE LAKES FARM, Newbury

BRIDGE ROAD LAKES, Warren Rondini, Middlefield

BROKEN BIT RANCH, Blanche Fleder, Garrettsville 
Geauga County (cont'd.)

LEDGEWOOD GAME FARM, INC., Louis

Scamecchia, Box 641, Parkman

HOSMER LAKES, Frank and Robert

Hosmer, Burton

LAKE FIORETTE, Rose Kirkpatrick, Chardon

NEUBAUER GAME FARM, Fritz

Neubauer, Route 1, Burton

ROSE FARM, Myron Rose, Russe11

SISSON ROAD LAKES, Joe Striet,

Route 1, Chardon

TROUT HAVEN, Robert Cornelius, Burton

WHITFORD WOODS, Windsor Ford,

Route 2, Middlefield

Greene County

HARLAN HURLEY FARM, Harlan

Hurley, Route 3, Xenia

\section{Guernsey County}

DON LUCAS LAKE, Don Lucas,

Route 2, New Concord

KIRKMAN BROTHERS PAY LAKE, Byesville

BICHARD PAY LAKE, Raymond

Bichard, Route 1, Kimbolt on

SPRING LAKE, Cambridge

WILLS CREEK, Coshocton

Hamilton County

BALDY'S FISHING LAKES, 5136 Foley, Cincinnati 38

HAMILTON COUNTY PARK, 1000 Miles Road, Cincinnati 31

HASKINS FISHING LAKE, 6664 Daly, Cincinnati

KRAMERS FISHING LAKE, $3340 \mathrm{~W}$. Galbraith, Cincinnati

LAKE CAST A LINE, Wards Corner Road, Lovel and

LAKE GLORIA, 10511 Pippin Road, Cincinnati 31 ,

LAKE NINA, 7200 Pippin Road, Cincinnati

SNOW SPRING LAKE, E. Priessman, Dry Ridge Road, Cincinnati

SPRINGDALE LAKE, 12117 Spring-

Field Pike, Cincinnati 46
Harrison County

HARRISON GUN CLUB, Harold Polen, Uhrichsville

Hocking County

CAMP BEUNA VISTA, Charles and

Lawrence Hines, Route 1 ,

Rockbridge

CAMP GREEN HILL, Route 3, Logan

COLUMBUS BEAGLE CLUB, Route 3,

Logan

HIDDEN LAKE, Ray Grossman,

Route 2, Logan

MATHIAS PARK, Harry Mathias,

Route 1, Rockbridge

Holmes County

O'DELLS LAKE, Big Prairie

TROUT PARK, Don Vavra, Glenmont

\section{Huron County}

PALMER LAKE, Wendell Palmer, Route 1, Greenwich

Jackson County

BAILEY LAKE, John Bailey,

Route 5, Jackson

RICHARDS PAY LAKE, Cecil

Richards, Route 2, Wellston

TRIPPIES LAKE, Chester Triplett, Route 1, Ray

VAN FOSSEN LAKE, Charles Van

Fossen, Route 3, Jackson

Jefferson County

AUSTIN LAKE, INC., Glenn Cable,

Route 1 , Irondale

BRANDYWINE LAKE, Donald W.

Damewood, Box 149, Toronto

FRED'S LAKE, Route 1, Richmond

GEORGE'S LAKE, Thomas George, Bergholz

MALABU LAKE, Donald and David

Pesta, 1205 Lincoln Ave.,

Steubenville

Lawrence County

BUFFALO LAKE, Buffalo Creek Road, Chesapeake 
Lawrence County (cont'd.) BURLINGTON LAKE, Route 2, South Point

CHUCKS LAKE, Charles Eicken, Coal Grove

CRESCENT LAKE, Mrs. Roberts, Route 7, Chesapeake

HOOTIN HOLLER, Hubert Frazier, Hanging Rock

LAKE FOREST, State Route 378, Chesapeake

LAWCO LAKE ASSOCIATION, Ironton PINE LAKE, Chesapeake

TWIN LAKES, Woodrow Bowman, Route 3, Pedro

\section{Licking County \\ CHAPMAN'S SUN VALLEY LAKE,} Kenneth Chapman, Dayton Road, Route 1, Newark

LAKE OTTO, Calvin Stockli, Route 3, Frazeysburg

\section{Logan County}

AL-KEN LAKES, K. J. Roberts, Route 2, Bellefontaine BRIGGS LAKE, E1don Briggs, Route, Bellefontaine

FISHING LAKE, R. E. Starbuck, Route 2, West Liberty

STONY CREEK TROUT \& PHEASANT CLUB, Don M. Hilliker, Route, DeGraff

\section{Lorain County}

KATINA LAKE FISHING CLUB, Mr. Chlepciak, 39421 Center Ridge Road, Elyria

\section{Mahoning County}

ARROWHEAD LAKE PARK, Fred Di11, Columbiana

\section{Medina County}

GRANGER LAKE, Route 2, Medina HILL N' DALE, INC., Gene Tyrre11, Medina
Meigs County

HIDDEN LAKES, Mrs. Harry Roush, Minnersville

MAPLEWOOD LAKE, Gene Hupp,

Route 1, Racine

PARSONS LAKE, James Parsons, Long Bottom

AULT FARM LAKE, Route 4, Woodsfield

ASHERS ${ }^{\prime} \frac{\text { Montgomery County }}{\text { FISHING LAKES, James }}$

Asher, Dechant \& Sulphur Springs

Road, New Lebanon

CRESTVIEW LAKE, Puddin Bag Road, Route 1, Germantown

DAYTON PLAZA FISHING LAKE, 2694 Wagoner Ford Road, Dayton 14

DIAMOND MILL LAKE, Frank Woxman, S. Diamond Mi11 Road, Route 2, New Lebanon

GEYSER LAKE, Washington Church Road, Miamisburg

LOLA'S FISHING LAKE, 2052 Va1ley Pike, Dayton 4

MACK'S LAKE, Mack Gilland, Crestway Drive, Clayton

NORTHRIDGE FISHING LAKE, 4599 North Dixie Drive, Dayton 14

RONDO LAKE, S. Diamond Mil1 Road, Route 2, New Lebanon

SPRINGBROOK LAKE, 2677 Soldiers Home, W. Carrollton Road, Dayton 18

SULPHUR GROVE FISHING HOLE, 7575

Taylorsville Road, Dayton 24

\section{Muskingum County}

DIEHR LAKE, Wa1ter Diehr, East Fultonham

LAKE ISABELLA, East Fultonham

LAKE VIEW, Robert Hopper, Pinkerton Lane, Route 2, Zanesville

Perry County

DAUGHERTY PAY LAKE, Joe B. Daugherty, Route 2, Somerset 
Perry County (cont'd.) MASTERSON'S, Roy A. Masterson, Route 2, Crooksville

\section{Pickaway County}

HILL LAKE, Mrs. J. B. Hill, Route 1, Orient

IVYWOOD LAKES, Cana1 Winchester LOOSE LAKE, C. H. Loose, Route 1, Orient

\section{Pike County}

LIMING LAKE, Delbert Liming, Route 5, Chillicothe

PINE LAKE, Lee Brown, Route 1, Waverly

WEAVER VACATION FARM, Harry Weaver, Route 3, Waverly

WYCKOFF PAY LAKE, Charles Wyckoff, Route 2, Piketon

\section{Portage County}

AURORA LAKE, Phil George, Box 91, Aurora

ELM LAKE, Streetsboro Township

LAKE JAY, Jay Ferry, Estworthy Road, Route 6, Ravenna

LAKESIDE SAND AND GRAVEL, Harry Kotkowski, 3501 Frost Road, Mantua

MARY'S LAKE, Herbert Reynolds, Di amond

MOGADORE LAKE, Akron

ROCK ISLAND, Dale Doak, 3227

Dawley Road, Ravenna

SHULTZ LAKE, 1052 Industry

Road, Atwater

SUNNY LAKE, Aurora Township

WHITTLESEY LAKE, Harry Whittlesey, Atwater

WINGFOOT LAKE, Akron

ANDERSON'S LAKE, N. E. Anderson, Route 2, New Paris

BERRY LAKES, Eaton Lewisburg Road, Lewisburg

DOC'S LAKE, Dr. E. D. Modlin, Route 4, Eaton
Preble County (cont'd.)

FRANCE PARK, New Paris

GROOMS LAKE, C. C. Grooms,

Route 2, New Paris

HADDIX FISHING LAKES, Charles

Haddix, West Elkton

LAZY LAKE, Roy Shock, Route 1, New Paris

LEWISBURG FISHING LAKES, Route 1, Lewisburg

MARVA LAKES, Dewey Walton,

Route 2, New Paris

ST. JACQUES LAKE, Chester Fudge, Eaton

SPRING LAKE, Omer Gratz, Route 1 , Pandora

\section{Ross County}

JERRY'S FISHING CLUB, Morris

Templin, Londonberry

KRETZER'S FISHING LAKE, Don

Kretzer, Potts Hill Road,

Bainbridge

LAKE HILL, Hil1 Bros., Frankfort

GILLUM LAKE, Richmondale

SUN VALLEY LAKE CLUB, John F.

Miller, Jr., 81 South Mulberry, Chillicothe

TYME LAKE, Idle Tyme, Route 2, Bainbridge

\section{Scioto County}

CLIFF' S LAKE, Arthur Clifford, Route 2, Sciotoville

LAKE MARGARET, Mrs. Margaret

Campbe11, Lucasville

LEWIS LAKE, Otto Lewis, Route 3, Wheelersburg

MEAD LAKE, T. G. Scott, Wheelersburg

RASE'S LAKE, Carl Rase, Minford SCOTT'S LAKE, C. W. Scott, Route 4, Luca

\section{Stark County}

CLAYS PARK, INC., Ottis Clay, Weygnt Road, Canal Fulton 
Stark County (cont'd.)

ECHO VALLEY FARM, Robertsville

HIDDEN VALLEY LAKE, Donald Cole,

Baird Road, Paris

LAKE-O-PINES, William Kimball,

1481 Lake-0-Pines Road,

Hartville

LAKE SHERMAN, Lake Drive, Navarre

PLEASANT LAKE PARK, Pleasant Lake Road, East Sparta

PLEASANT VALLEY LAKE, Tony

Stefanick, Route 1, East Canton WILLOW SPRINGS, 1715 - 55th St.,

N. W., Canton

\section{Summit County}

CRYSTAL LAKE PARK, 0. Clare Conlan, Crystal Lake Road, Akron

FELL LAKE PARK, Tony Perko, 10070 Akron-Cleveland Road, Northfield

HUDSON SPRINGS PARK, Michael C. May, 15 Baldwin, Hudson

LAZY LAKE, Michae1 Korba, 140 Virginia Kendall Road, Peninsula

LOYAL OAK LAKE PARK, Robert Emery, 2678 S. Hametown Road, Barberton

REVERE LAKE, Harley Boltz, 2213 Revere Road, Akron 13

WHITE POND BEACH, Galliano Martin, 2113 Copley Road, Akron 20

WYOGO LAKE PARK, James A. Loftus, Jr., 4419 Wyoga Road, Stow

CAMP GREENACRES, Frank

Cunningham, Route 1, Farmdale

CAROMA RANCH, Mark Hall, Route 1 , Lockwood

HAWTHORNE LAKE, Don Rodney, Route 1, Bristolville
Tuscarawas County

DEVILS DEN PARK, N. Storad, Route 2, Tippecanoe

PARADISE LAKES, H. Horsefall,

Route 3, New Philadelphia

Union County

TWIN LAKES, Grover Ellis, Route 3, Msrysville

\section{Vinton County}

OWSLEY PAY LAKE, William Owsley, Route 2, McArthur

PECK PAY LAKE, Willard Peck, McArthur

YOUNG PAY LAKE, William Young, Hamden

ZINN PAY LAKE, Ernest Zinn, Hamden

ARMCO ASSOCIATION PARK, Route 3, Lebanon

CAMP BUTTERWORTH, Route 1, Maineville

CAMP FIREFLY, Route 1, Oregonia

CAMP JOY, Rev. Morris McCrackin, Route 1, Clarksville

CAMP STONYBROOK, Waynesville

CAMP WA-KA-KEE, Route 1 , Oregonia

CHURCH OF GOD YOUTH CAMP, Rev. Steenbargen, Route 1, Oregonia

CRAIG KENNELS, Lou Craig, Route 1, Lebanon

GINE-BELL LAKE, Route 1, Morrow

GREEN ACRES FISHING LAKE, Landis Whitt, Route 1, Lebanon

LILLY LAKE, Randall Lilly, Morrow

PINE HILL LAKE, G. K. Howard, 211 Kings Mill Road, Mason

PINEY WOODS, A. E. Switzer, Route 1, Morrow

SNIDER LAKE, Ernest Snider, Route 1, Maineville

SPRING VALLEY WILDLIFE AREA, spring Valley 
Warren County (cont'd.)

WILLOW LAKES, Route 2, Morrow

WUNKER'S LAKE, R. B. Wunker,

Shadow Lake

Washington County

LAKESIDE, Chop U11man, Beverly

SECOY LAKE, Carl Secoy, Barlett

SIEDA, Carl L. Weppler and Albert

Berg, Route 2, Whipple

Wayne County

CASKEY LAKE, Donald Caskey,

Route 1, Orrville

PRAIRIE LANE SHOOTING PRESERVE, Arthur Miller, Route 4, Wooster

ROHR PARKS, John Rohrer, Route, Doylestown

\section{Williams County}

BORTON FARM POND, Ray Borton, Route 1, West Unity

DREAMLAND ACRES, Edward Brodbeck,

Route 1, Montpelier

Wyandot County

CAREY SPORTSMEN CLUB, Carey

RIVER VALLEY SPORTSMENS CLUB, Upper Sandusky

TEXAS TOWNSHIP SPORTSMEN CLUB,

Syc amore

WALTON LAKE, Sam Walton,

Upper Sandusky

WYANDOT ROD \& GUN CLUB, Route 1,

Upper Sandusky

WYANDOT SPORTSMEN CLUB, Upper

Sandusky 


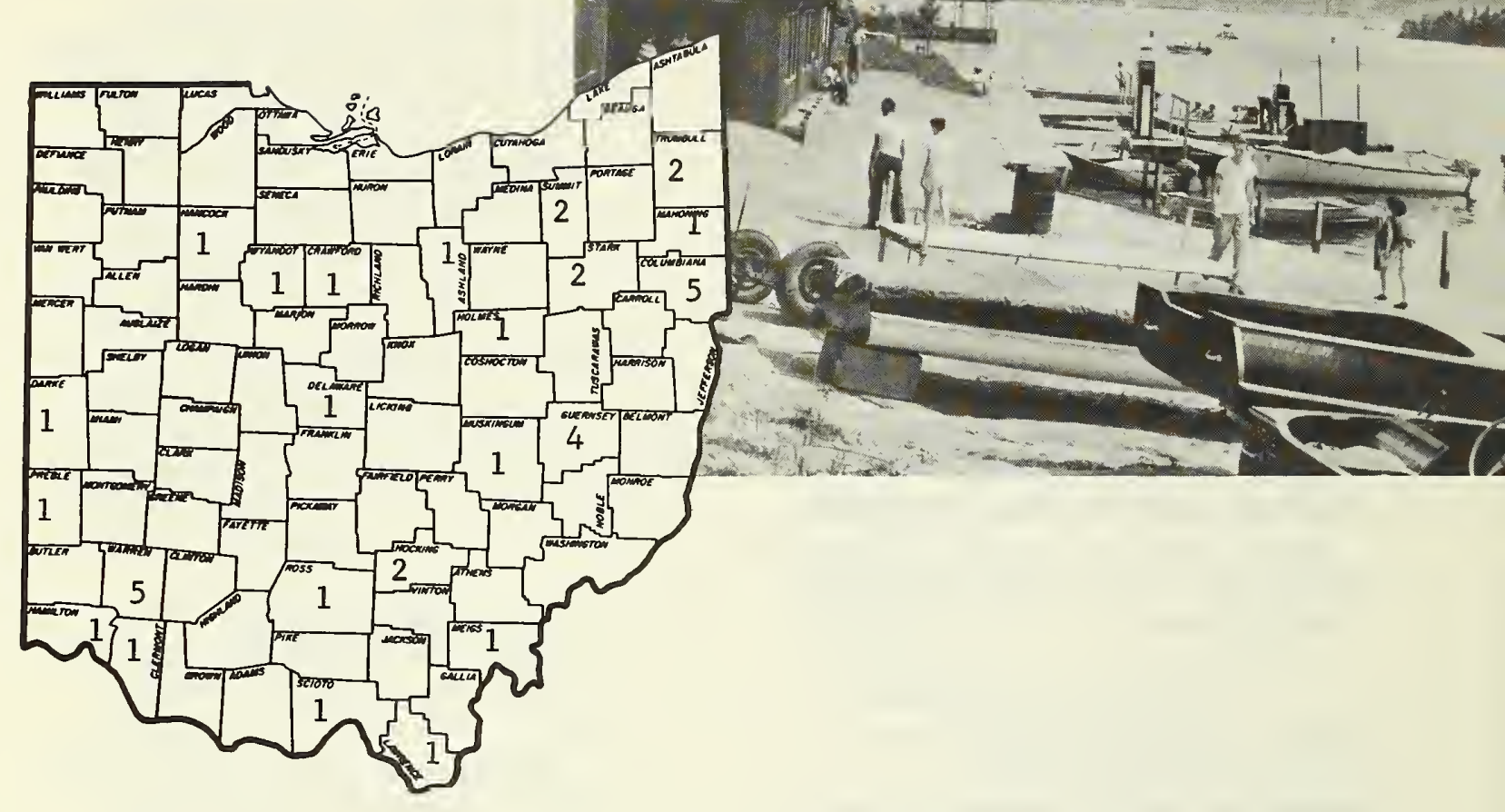

\section{BOAT RENTALS}

Boats may sometimes be rented at pay lakes. But in some places in Ohio, boats may be rented for purposes other than fishing such as for canoeing along wooded streams. Boat docking, outboard motor rental, and sales of gasoline and oil are examples of other services that may be provided. Boats are usually rented by the hour or by the day.

\section{Ashland County}

NEUBRANDER'S, Art Neubrander,

Route 1, Sullivan

\section{Clermont County}

RAPP'S CAMP GROUND, Route 1,

U. S. Highway 52, Felicity

\section{Columbiana County}

CONSER RUN, Theodore and Kent

Sanor, Route 1, East Rochester JENKINS LAKE, Sterling Jenkins,

Route 1, Salineville

LAKE CHA-VEL, David Tice,

Route 1, Wellsville
Columbiana County (cont'd.) TERRYS LAKE CORP., Willard Stol1, Route 2, Lisbon

WESTVILLE LAKE, Allen Craven, Westville

\section{Crawford County SUNSET SPRINGS, George Preston, Oceola}

\section{Darke County}

WAYNE LAKES, Lowe11 Vietor, Route 1, Greenville 
Delaware County

HOLIDAY HILL, John Rowlands, Route 3, Delaware

Guernsey County

DON LUCAS LAKE, Don Lucas, Route 2, New Concord

KIRKMAN BROTHERS PAY LAKE, Byesville

BICHARD PAY LAKE, Raymond Bichard, Route 1, Kimbolton

SPRING LAKE, Cambridge

Hamilton County

HAMILTON COUNTY PARK, 1000 Miles Road, Cincinnati 31

Hancock County

BENROTH RECREATION CLUB, Z. M. Benroth, Route 5, Findlay

\section{Hocking County}

CAMP ALLEGRO, Robert White, Route 2, Logan

CAMP GREEN HILL, Route 3, Logan

O'DELLS LAKE, Big Prairie

Lawrence County

LAKE FOREST, State Route 378, Ches apeake

\section{Mahoning County}

ARROWHEAD LAKE PARK, Fred Di11, Columbiana

Meigs County

MAPLEWOOD LAKE, Gene Hupp, Route 1, Racine

Muskingum County

LAKE ISABELLA, East Fultonham

$$
\text { Preble County }
$$

Ross County

ATER'S LAKE, Raymond Ater, Route 3, Chillicothe

\section{Scioto County}

LAKE MARGARET, Mrs . Margaret Campbe11, Lucasville

Stark County

CLAYS PARK, INC., Ottis Clay, Weygnt Road, Canal Fulton

CRYSTAL LAKE PARK, 4848 Crystle Lake Road, Massillon

\section{Summit County}

FELL LAKE PARK, Tony Perko, 10070 Akron-Cleveland Road, Northfield

HUDSON SPRINGS PARK, Michael C. May, 15 Baldwin, Hudson

\section{Trumbu11 County}

CAROMA RANCH, Mark Ha11, Route 1, Lockwood

HAWTHORNE LAKE, Don Rodney, Route 1, Bristolville

ARMCO ASSOCIATION PARK, Route 3, Lebanon

CAMP HOOK, Route 1, Oregonia

CAMP JOY, Rev. Morris McCrackin, Route 1, Clarksville

CAMP SWANEKY, Route 1, Oregonia

CAMP WHIP-OR-WILL, Route 1 , Morrow

\section{Wyandot County \\ WALTON LAKE, Sam Walton, Upper Sandusky}

FRANCE PARK, New Paris 


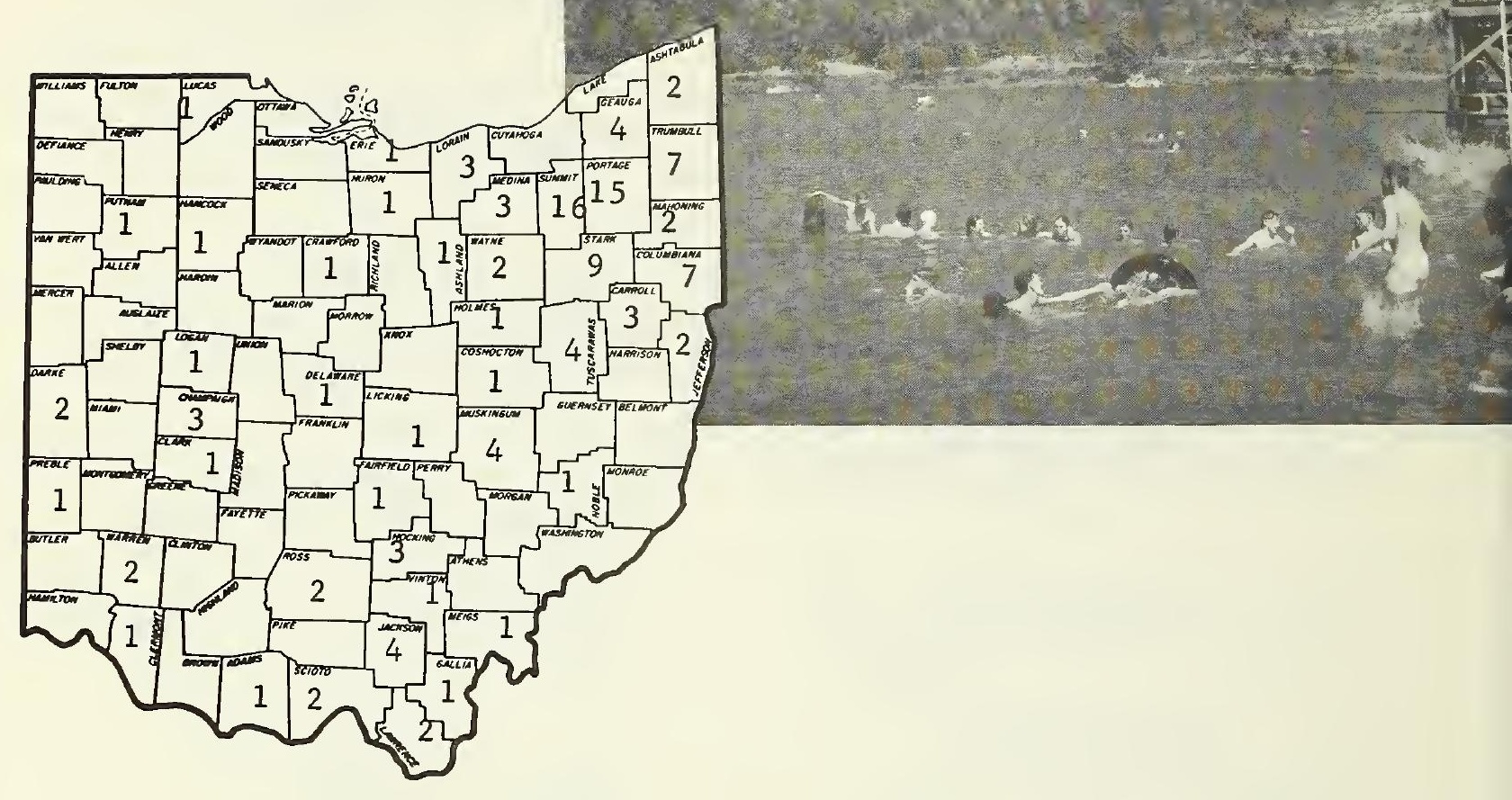

\section{PRIVATE BEACHES}

Beaches on private lakes or ponds were the only swimming areas included in the inventory. The degree of development of swimming areas ranges from farm ponds having no improvements to those on large lakes with sanded beaches, diving boards, bathhouses, etc. Beaches are often bordered by woodlands in which picnic tables and other facilities are available. Private beaches are also often a part of other recreation enterprises such as pay 1 akes and campgrounds. An entrance fee is usually charged and equipment may be rented. However, a weekly, monthly or yearly membership may be required.

\author{
Adams County \\ WREN'S PARK, C. D. Wren, \\ West Union
}

\section{Ashland County}

NEUBRANDER'S, Art Neubrander, Route 1, Sullivan

\section{Ashtabula County}

BUCK LAKE, Conneaut

\section{Ashtabula County (cont'd.)}

LAZY ACRES, R. E. Butler, Route 2, Geneva

\section{Carrol1 County}

BILLY B. BEACH, Ruth Blackstone, Route 1, Carrollton

FOLTZ LAKE, James Foltz, Malvern VO-ASH LAKE, Steve Neggett, Route 3, Carrollton 
Champaign County

BALI HIGH, Robert Randa11,

Route 2, Urbana

LAKEWOOD BEACH, Herbert McBride, Route 4, Urbana

MUZZY LAKE AQUATIC CLUB, Urbana

Clark County

ENON ROAD BEACH CLUB, Route 1, Springfield

\section{Clermont County}

RAPP'S CAMP GROUND, Route 1,

U. S. Highway 52, Felicity

Columbiana County

COPELAND LAKE, W. L. Vaughn,

Leetonia

DILL LAKE, Fred Dil1, Columbiana JENKINS LAKE, Sterling Jenkins,

Route 1, Salineville

LITTLE ROCK, Charles Bibbee,

Route 1, East Liverpool

MAR-VIN LAKE, F. D. Irwin, Radio

Heights, East Liverpool

QUEENS GARDEN, Paul Glunt,

Route 1, Hanoverton

WESTVILLE LAKE, Allen Craven, Westville

Coshocton County

BARTH LAKE, Fred Barth, Route 2, Fresno

SUNSET SPRINGS, George Preston,
Oceola

Darke County

STILLWATER BEACH, Route 1, Bradford

WAYNE LAKES, Lowe11 Vietor, Route 1, Greenville

Delaware County

ECKLES LAKE, L. E. Eckles, 914 Pollock Road, Delaware
Erie County

ANDERSON ACRES, INC., Hurop

\section{Fairfield County}

TIKI CLUB, Richard Byrd, Route 5, Lancaster

\section{Gallia County}

CAMP ASBURG, Portsmouth

\section{Geauga County}

HOSMER LAKES, Frank and Robert Hosmer, Burton

OCEANA PARK, M. Matrai, Route 1, Burton

ROSE FARM, Myron Rose, Russe11

SPRING LAKE PARK, Manny Fendrich, East Claridon

Hancock County

BLUE PIGEON, Cletis Winstead, Route 5, Findlay

Hocking County

BIG BROTHERS CAMP, Route 2, Logan

CAMP AKITA, Route 2, Logan

CAMP ALLEGRO, Robert White, Route 2, Logan

Holmes County

O'DELLS LAKE, Big Prairie

Huron County

FENNWOOD HUNT CLUB, Robert Fenn, Route 1, Bellevue

\section{Jackson County}

BAILEY LAKE, John Bailey, Route 5, Jackson

RICHARDS PAY LAKE, Cecil Richards, Route 2, Wellston

TRIPPIES LAKE, Chester Triplett, Route 1, Ray

VAN FOSSEN LAKE, Charles Van

Fossen, Route 3, Jackson 
Jefferson County

AUSTIN LAKE, INC., Glenn Cable, Route 1 , Irondale

GEORGE'S LAKE, Thomas George, Bergholz

\section{Lawrence County \\ GINA LAKE, Coal Grove \\ LAKE FOREST, State Route 378, Ches apeake}

\section{Licking County}

LAKE OTTO, Calvin Stock1i,

Route 3, Frazeysburg

\section{Logan County}

AL-KEN LAKES, K. J. Roberts, Route 2, Bellefontaine

\section{Lorain County}

BONFADINI'S, John Bonfadini, Columbia Station

GILGENBACH'S, Gletus Gilgenbach, Route 3, Elyria

SPRING LAKE PARK, Mr. Gerber, Amherst

Lucas County

CENTENNIAL RECREATION CLUB, 1943 Centennial Ave., Toledo

\section{Mahoning County}

ARROWHEAD LAKE PARK, Fred Dill, Columbiana

WILDWOOD LAKE PARK, Carl Shank, North Lima

\footnotetext{
Medina County

GRANGER LAKE, Route 2, Medina

HILL N' DALE, INC., Gene Tyrre11, Medina

OSAGE LAKE PARK, Roy Lutz, Wadsworth
}

Meigs County

MAPLEWOOD LAKE, Gene Hupp, Route 1, Racine
Muskingum County

DIEHR LAKE, Walter Diehr, East Ful tonham

LAKE ISABELLA, East Fultanham

LAKE VIEW, Robert Hopper, Pinkerton Lane, Route 2, Zanesville

NORTHSIDE BEACH, Larry Finan, Frazeysburg Road, Zanesville

Noble County

EAGLES CLUB FARM, Ernest Stritz, Caldwe 11

DUNCANSIDE, M. B. Duncan, 549 Howe, Kent

EDINBURG BEACH CLUB, Mrs. George Conobre, New Mil ford

FRIENDSHIP ACRES PARK, Randolph HICKORY HILL PARK, Wallace

Girton, New Milford

HOLIDAY SANDS, Arthur Klohn,

State Route 14, Ravenna

KALBAUGH LAKE, Ralph Kalbaugh, 3848 Sunny Brook Road, Kent

LAKE JAY, Jay Ferry, Estworthy Road, Route 6, Ravenna

MARY'S LAKE, Herbert Reynolds, Diamond

MATHEW NOAH LAKE, Mathew Noah

PLAY LAND, State Route 224, Randolph

ROCK ISLAND, Dale Doak, 3227 Dawley Road, Ravenna

ROLLING ACRES BEACH CLUB, Don Chamberlin, 7814 Infirmary Road, Ravenna

ROUND-UP LAKE PARK, Mr. Ballonoff, Mantua

WHITTLESEY LAKE, Harry Whittlesey, Atwater

WILLOW BEACH CLUB

FRANCE PARK, New Paris 
Putnam County

SPRING LAKE, Omer Gratz, Route 1 , Pandora

\section{Ross County \\ ATER'S LAKE, Raymond Ater, Route 3, Chillicothe \\ LAKE HILL, Hill Brothers, Frankfort}

\section{Scioto County}

LAKE MARGARET, Mrs. Margaret

Campbe11, Lucasville

LEWIS LAKE, Otto Lewis, Route 3, Wheelersburg

\section{Stark County}

CLEARWATER PARK, Robert Hostetler, Edison St., Hartville

HIDDEN VALLEY LAKE, Donald Cole, Baird Road, Paris

HIGH MILL PARK, Mrs. Sanders, High Mill Ave., Massillon

LAKE SHERMAN, Lake Drive, Navarre

MENEGAY'S PARK, Richard Menegay, Louisville

MUZZY LAKE, James Muzzy, 830 - 38th N. W., Canton

PLEASANT LAKE PARK, East Sparta STARRY HI, INC., O. F. Bairer, 2664 Belfort Ave., Louisville SUNSET PARK, Robert Hostetter, Edison St., Alliance

\section{Summit County \\ BOBS LAKE, Curt Sarff, 4936 S. Arlington St., North Canton 20}

CARTER LAKES, Harold Carter, 9032 Charles Court, Twinsburg

CRYSTAL LAKE PARK, O. Clare Conlan, Crystal Lake Road, Akron

FELL LAKE PARK, Tony Perko, 10070 Akron-Cleveland Road, Northfield
Summit County (cont'd.)

HOLIDAY PARK, Alexander Plata, 9610 Chamberlin Road, Twinsburg

HUDSON SPRINGS PARK, Michael C. May, 15 Baldwin, Hudøon

KOOL LAKE PARK, George Kuli, 1043 Hardy Road, Cuyahoga Falls LAKE KIM-TAM, C. W. Patton, 194 Praire Drive, Akron 12 LOYAL OAK LAKE PARK, Robert Emery, 2678 S. Hametown Road, Barberton

MACA PARK, Irwin and Don Bowsher, 988 Beechwood Drive, Tallmadge

MUNROE FALLS PARK, Jack Renner, Munroe Falls

SILVER SPRINGS PARK, Russel1 Karas, 5027 Stow Road, Stow TALLMADGE SPRINGS PARK, INC., Dennis Barnes, 619 Northwest Ave., Tallmadge

TAMSIN PARK, Route 1, Peninsula WHITE POND BEACH, Galliano Martin, 2113 Copley Road, Akron 20

WYOGO LAKE PARK, James A. Loftus, Jr., 4419 Wyogo Road, Stow

ANDERSON'S LAKE, Robert G. Anderson, Route 1, Vienna

CAMP GREENACRES, Frank Cunningham, Route 1, Farmdale

CAROMA RANCH, Mark Hal1, Route 1, Lockwood

FARMER JIM'S, B. A. Callahan, 1610 Niles-Cortland Road, Cort 1 and

SMELKO'S, Al Smelko, 1955 NilesCortland Road, Cortland

SPRING LAKE, Fred Newberry, Bristolville

WILLOW LAKE PARK CLUB, Nick Dan, 6863 Mahoning N. W., Warren

Tuscarawas County

DEVILS DEN PARK, N. Storad, Route 2, Tippecanoe 
Tuscarawas County (cont'd.)

PARADISE LAKES, H. Horsefall,

Route 3, New Philadelphia

RAINBOW LAKES, Nick Pappas,

Route 1, Newcomerstown

TALL TTMBER LAKE, J. Turkovich, Route 1, New Philadelphia

Vinton County

COLUMBUS COMMUNITY COUNCIL,

Thelma Stinson, New Plymouth

Warren County

CAMP WA-KA-KEE, Route 1 , Oregonia

PINE HILL LAKE, G. K. Howard, 211 Kings Mill Road, Mason

Wayne County

HOMAN'S RECREATION, Gene Homan, Route 1, Marshallville

ROHR PARKS, John Rohrer, Route, Doylestown 


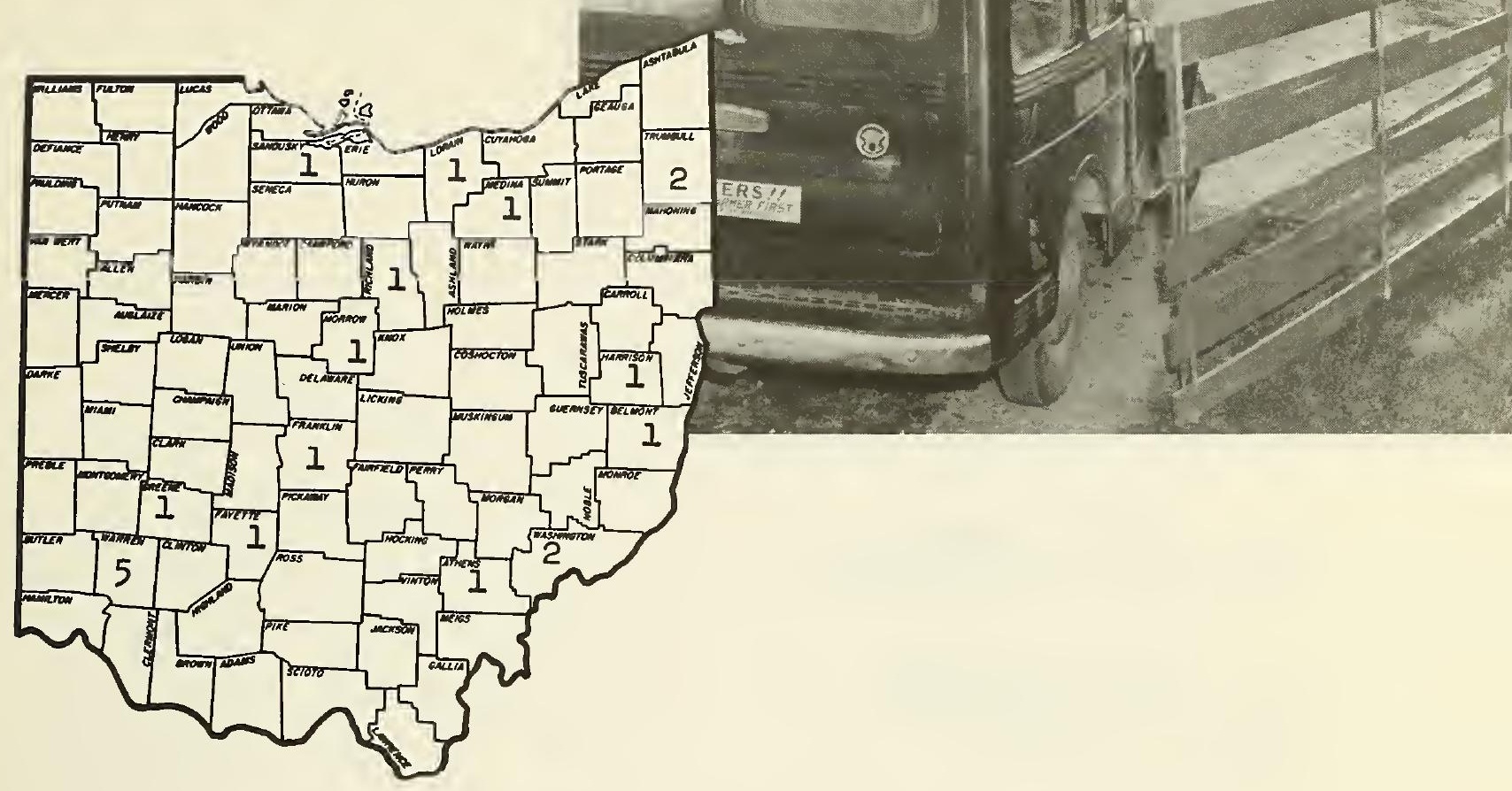

\section{GENERAL HUNTING AREAS}

General hunting areas are either woodlands, brushlands, or farmlands with sufficient food and cover for game to flourish. The type of game available varies with the food and cover on the land. Most operators provide for rabbit, pheasant, and quail hunting, but woodland owners frequently provide for squirrel and raccoon hunting. Where ponds and lakes are suitably located, duck hunting may be available. The charge is usually an entrance fee for hunting with additional fees for other services that may be furnished, such as hunting and retrieving dogs.

\section{Athens County \\ HANNING'S LAKE, Alton Hanning, Route 5, Athens}

Belmont County

CAPTINA WILDLIFE ASSOCIATION, Ralph Carpenter, Somerton

\section{Fayette County} BROWNING FARM, John Browning, Route 5, Washington Court House

\section{Franklin County}

VALLEY RANCH PICNIC AREA, Fletcher Audrix, 8802 W. Broad St., Columbus

\section{Greene County}

HARLAN HURLEY FARM, Harlan Hurley, Route 3, Xenia

\section{Harrison County}

JEWETT SPORTSMAN CLUB, Max Mickey, Route, Jewett 
Lorain County

WASSERMAN PLACE, S. L. Was serman,

Route 1, Oberlin

\author{
Medina County \\ HILL N' DALE, INC., Gene Tyrre11, \\ Medina
}

\author{
Morrow County \\ BEAGLE CLUB, Galion \\ Richl and county \\ RICHLAND COUNTY COONHUNTERS \\ ASSOCIATION, Sherman Henry, \\ Route 3, Mansfield \\ Sandusky County \\ SUGAR CREEK PROTECTORS GAME \\ ASSOCIATION, Gibsonburg \\ Trumbu11 County \\ CAROMA RANCH, Mark Hal1, \\ Route 1, Lockwood \\ WINDHAM HUNTING AREA, Warren \\ Warren County \\ COCHRAN HUNTING AREA, Melville \\ A. Cochran, Route 1, Morrow \\ CRAIG KENNELS, Lou Craig, \\ Route 1, Lebanon \\ LILLY LAKE, Randall Lilly, \\ Morrow \\ SNIDER LAKE, Ernest Snider, \\ Route 1, Maineville \\ SPRING VALLEY WILDLIFE AREA, \\ Spring Valley \\ Washington County \\ LAKESIDE, Chop U1lman, Beverly \\ SECOY LAKE, Carl Secoy, Barlett
}


Allen County

* THE FORT AMANDA CLUB, Route 4, Lima

Ashtabula County

* CANNING SHOOTING PRESERVE, Henry Canning, Creek Road, Jefferson SAYBROOK PHEASANT SHOOTING PRESERVE, 4746 Main Ave., Ashtabula

WILLIAMS SHOOTING PRESERVE, Bob Williams, Route 2, Rome

\section{Brown County}

* ST. MARTiNS ASSOCIATION, Fayet teville

Butler County

* FINCASTle FARMS, 1.906 Highland Ave., Cincinnati 19

\section{Carroll County}

* DONAHEY SHOOTING PRESERVE, Robert F. Donahey, 1436 Union Commerce Building, Cleveland 14

\section{Champaign County}

* VAN DARBY CLUB, Route 2, Mechanicsburg

\section{Clermont County}

* SUgar hiLl GUN CLUB, Box 599, Cincinnati 1

* TODD SHOOTING PRESERVE, Hawley Todd, Ward's Corner Road, Box 14, Miamiville

\section{Clinton County}

CHERRYBEND PHEASANT FARM, Route 4, Wilmington

Columbiana County

* THE CROCKERY CITY I \& P COMPANY, Route 2, Box 124, Lisbon

* VALley VIEW huNT ClUB, Route 4, Salem
Delaware County

* LAKE HILL LODGE, INC., 85 E. Gay St., Columbus

\section{Erie County}

* CASTALIA FARMS, INC., Box 337, Castalia

* OLD MILLSITE FARM, Route 1, Box 62, Castalia

Fairfield County

* BUCKEYE GAME CLUB, Route 3, Thornville

\section{Fayette County}

* BLUE FIELD HUNT CLUB 非, Route 1, Greenfield

FAYETTE HUNT AND GUN CLUB,

Route 5, Washington Court House

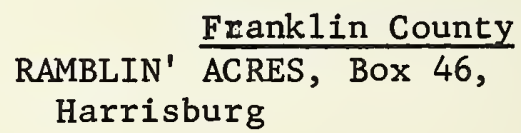

Geauga County

INDIAN SPRINGS PRESERVE, 1132 Ranchland Drive, Clevel and 24

* LEDGEWOOD GAME FARM, INC., Route 2, Garrettsville

* NEUBAUER GAME FARM, Fritz Neubauer, Route 1, Box 492, Burton

* WHITFORD WOODS, Route 2, Middlefield

* BLUE FIELD HUNT CLUB 非, Cedarville

STONE ROAD PHEASANT FARM, Stone Road, Route 2, Xenia

Hancock County

DRAY SHOOTING PRESERVE, James

Dray, Route 1, Bluffton

* engineERS CLUB, Box 450, Findlay

Huron County

* FENNWOOD HUNT CLUB, Route 1, Bellevue 
Huron County (cont'd.)

* PINECREST SHOOTING PRESERVE, Oberlin Road, Route 2, Elyria

\section{Licking County}

Mc ROBB FARMS, INC., Route 2, Utica

Lorain County

* B C LAND COMPANY, Box 5000, Clevel and 1

* BEAVER CREEK HUNT CLUB, CooperFoster Park Road, Amherst

* INDIAN HOLLOW LAKE HUNT CLUB, Indian Hollow Road, Route 2, Grafton

NIMROD CLUB, INC., BOX 636, Elyria

Lucas County

* CEDAR point CLUB, 1300 Leader Building, Cleveland 14

* DeWITT COMMERCIAL SHOOTING PRESERVE, Missionary Island, Waterville

N. P. SHOOTING PRESERVE, Genoa

* otTAWA SHOOTING CLUB, Eagle Point, Rossford

Medina County

* HILL N' DALE, INC., Route 7, Box 74, Medina

HOUND \& PHEASANT PRESERVE,

Route 2, Medina

Miami County

DAYTON SHOOTING PRESERVE,

4167 Upham Road, Dayton 29

* tAYLOR SHOOting PRESERVE, John N. Taylor, 214 S. Wilkinson St., Dayton

Ottawa County

* tuRTLE cREEK GUN CLUB, 300 Union Commerce Building, Cleveland 18
Portage County

EAGLE CREEK SHOOTING PRESERVE, Silica-Sand Road, Garrettsville

Ross County

SCIOTO GAME PRESERVE, c/o FOX

Paper Company, Cincinnati 15

* GIBSONBURG CLUB COMPANY,

1230 Illuminating Building, Cleveland 13

THE L. \& M. SHOOTING PRESERVE, c/o Lawrence Chambers, Genoa

* otTAWA SHOOTING CLUB, Route 5, Fremont

* WOODVILLE SHOOTING CLUB, INC., Box 218, Woodville

\section{Shelby County}

* BUNKER-HILL PRESERVE, INC., 109 N. Main Ave., Sidney

Stark County

* MUDBROOK GAME \& FISH ASSOCIATION, 6553 Hills \& Dales Road,

N. W. Canton 8

* NAVARRE SHOOting PRESERVE, Box 515, Massillon

Summit County

* SWDOHAHA FIELD CLUB, 2985 Montgomery Road, Shaker Heights, Cleveland 22

Trumbul1 County

* bear creek game CLUB, INC., Girdle Road, West Farmington

* LEDGEWOOD GAME FARM, INC., Box 641, Parkman

CENTRAL OHIO PHEASANT HUNTERS, Route 4, Marysville

Warren County

CAIRN COVERT, Route 1, Morrow

* LAND $0^{\prime}$ LAKES, Morrow 
Warren County (cont'd.)

* SAN MAR GALE SHOOTING PRESERVE, Morrow-Waynesville Road, Oregonia

Wayne County

PRAIRIE LANE SHOOTING PRESERVE, Route 4, Wooster

TALLMADGE PHEASANT FARM \& SHOOTING PRESERVE, Route 2, Jeromesville

Wood County

* BELMONT GUN CLUB, 931 National

Bank Building, Toledo 

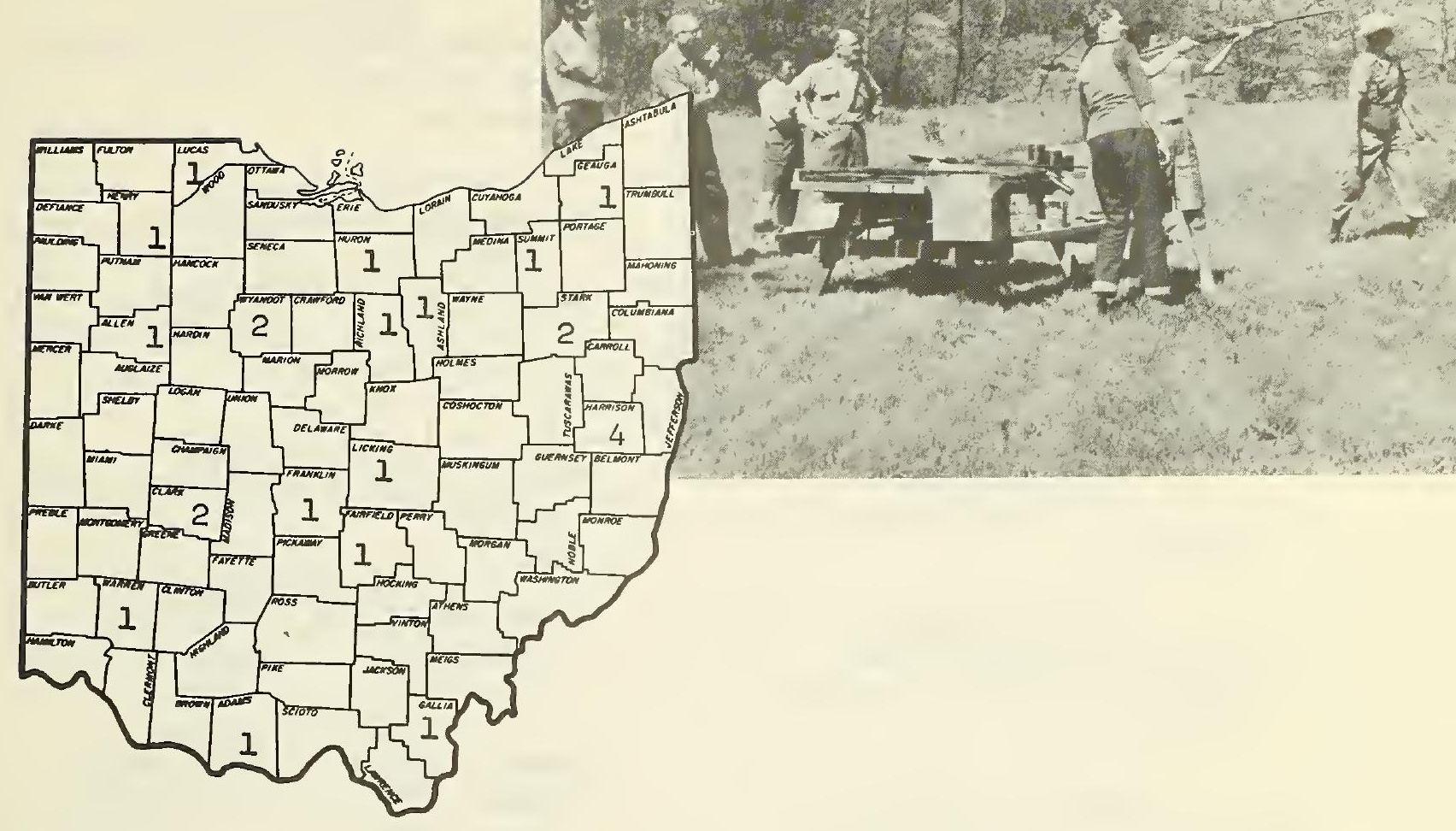

\section{SHOOTING AREAS}

Shooting areas may be operated by a gun club, or associated with a shooting preserve or hunting area. The three most common activities are target shooting, trap shooting, and skeet. Target shooting consists of practice firing at a butt or mark. Trap shooting consists of shooting at liberated pigeons or at glass balls and clay pigeons sprung into the air from a trap. Skeet is a form of trap shooting in which clay targets are thrown in such a way as to duplicate the angles of flight of birds and fowl. Picnicking and other facilities are generally provided in a forest setting. An entrance fee is usually charged with additional fees for supplies and equipment rental.

Adams County

ADAMS COUNTY GUN CLUB, N. B. Lindamood, Peebles

\section{Allen County}

THE FORT AMANDA CLUB, Norman Amstutz, 200 W. Market St., Lima

\section{Ashland County}

NEUBRANDER'S, Art Neubrander, Route 1, Sullivan

\section{Clark County}

SHAWNEE SKEET CLUB, Ed Buchwalter III, Plattsburg Lond Road, Route 1, South Charleston 
Clark County (cont'd.) SPORTSMAN CLUB, Ballentine Road, Route 2, Springfield

Fairfield County ADENA RIDGE, William Burnside, Route 1, Lancaster

Franklin County

EL RANCHO GUN CLUB, Myron Gifford and Carl Walker, 141 Doherty, Columbus 4

Gallia County GALLIPOLIS GUN CLUB, Route 2, Bidwe11

Geauga County LEDGEWOOD GAME FARM, INC., Louis Scamecchia, Box 641, Parkman

\footnotetext{
HAMILTON'S, Albert Hamil ton, Route 2, Freeport

HARRISON GUN CLUB, Harold Polen, Uhrichsville

JEWETT SPORTSMAN CLUB, Max Mickey, Jewett

SCIO SPORTSMAN CLUB, Roy Gatts, Scio
}

\footnotetext{
Henry County

HENRY COUNTY SPORTSMEN'S CLUB, Liberty Center

Huron County

FENNWOOD HUNT CLUB, Robert Fenn, Route 1, Bellevue

Licking County

DAR-LEE SHOOTING RANGE, 388 Taylor Road, Summit Station

Lucas County

TOLEDO GUN CLUB, Central Ave., Toledo
}

Richland County

RICHLAND COUNTY FISH \& GAME CLUB, William Stahl, Box 272, Mansfield

\section{Stark County}

SANDY VALLEY OUTDOOR LEAGUE TRAPSHOOT, East Sparta

SHELL \& GUN ARCHERY FARM, D. P. Estep, $10071 \mathrm{Cleveland}$ Ave., Greentown

\section{Summit County}

BUCKEYE SPORTS CENTER, 4610 AkronCleveland Road, Peninsula

\author{
Warren County \\ KINGS MILLS GUN CLUB, Columbia \\ Road, Route 1, Mason \\ Wyandot County \\ CAREY SPORTSMEN CLUB, Carey \\ WYANDOT ROD \& GUN CLUB, Route 1, \\ Upper Sandusky
}




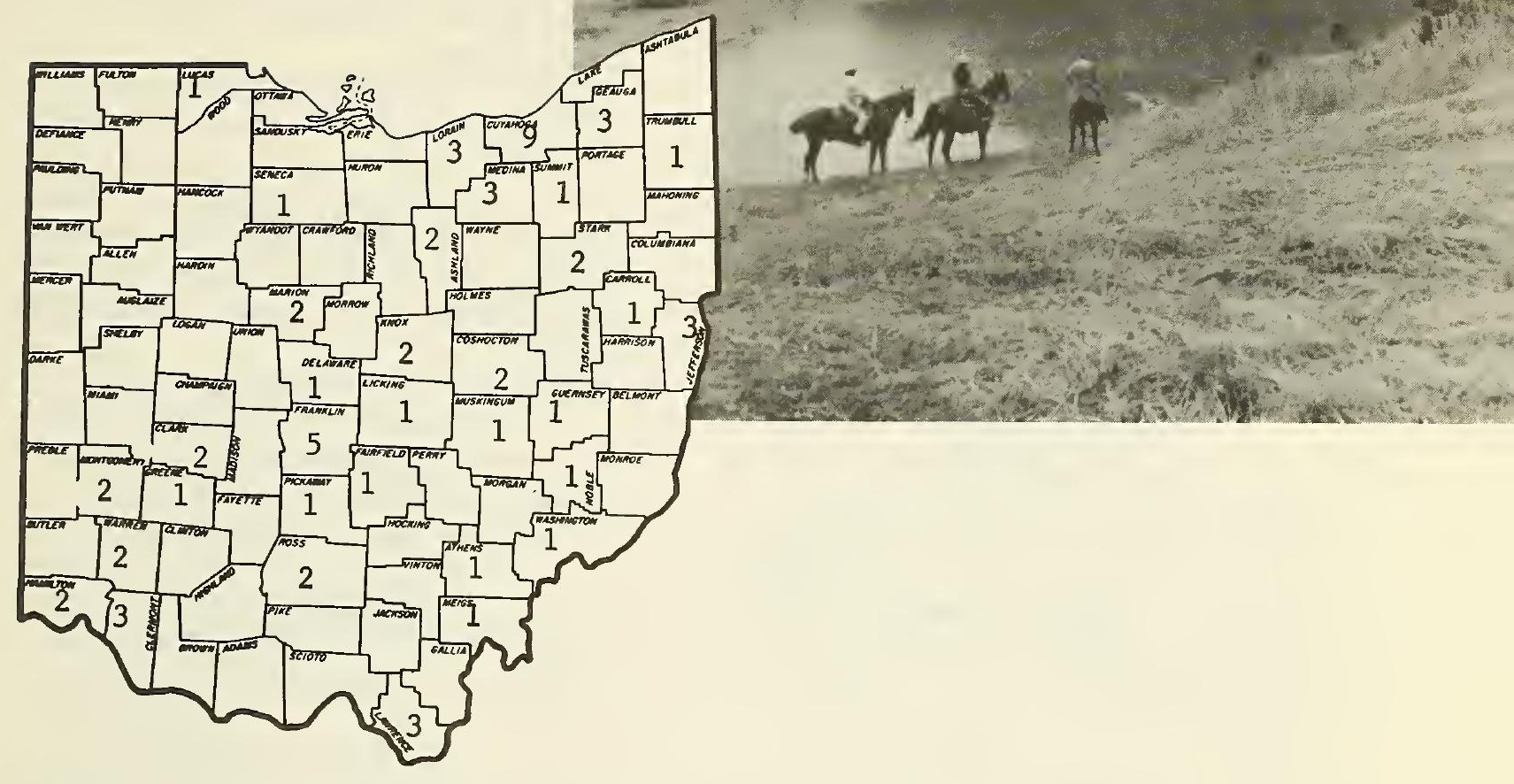

\section{RIDING STABLES}

Riding stables provide several activities revolving around horses or ponies. In summer, horseback riding, pony riding, and riding instructions are the main attractions. In fall and winter when weather is suitable, hay rides and sleigh rides are in order. Riding trails usually are routed along streams and through the woods and forest. The fee for horseback riding is usually on an hourly basis. Other services such as training and boarding of horses and ponies are often provided. Many riding stables are associated with vacation farms or dude ranches.

Ashland County

NEUBRANDER'S, Art Neubrander,

Route 1, Sullivan

STRAIT RIDING STABLE, Dr. John

Strait, Route 4, Ashland

\section{Athens County}

HANNING' S LAKE, Alton Hanning,

Route 5, Athens

\section{Carrol1 County}

CAMP FALCON, William V. Lorimer, Route 3, Carrollton

\section{Clark County}

BAR X RIDING STABLE, Burleigh Grimes, East National Road, Mounted Route 52, Springfield GRAY'S RIDING STABLE, Howard Gray, Titus Road, Route 5, Springfield 
Clermont County

CLOVER KNOLL RIDING \& DRIVING

ACADEMY, Route 2, Batavia

POST \& PADDOCK STABLE, 4519

Tealtown Road, Milford

RED FOX STABLES, INC., Sugar Camp

Road, Milford

\section{Coshocton County}

COCHRAN RIDING STABLES, Robert

Cochran, Route 2, Coshocton

MONTE VISTA STABLES, Harold

McClure, Route 1, Coshocton

\section{Cuyahoga County}

CLAGUE SADDLE \& BRIDLE CLUB,

4750 Tiedeman Road, Clevel and 9

DONOFRIO'S BOARD \& SALES STABLES,

626 West Bagley, Berea

FIVE A \& A STABLES, 3268 Edgerton,

Cleveland 18

LAKEWOOD RIDING STABLES, INC., 19901 Puritas, Clevel and 35

ROCKY RIVER STABLES, 20900 Hillside Road, Cleveland

SOMCREST STABLES CO., 380 SOM

Center Road, Cleveland

TRAIL O'PINES RIDING ACADEMY, SOM Center Road, Cleveland

TRIO FARM, 6949 Chagrin River Road,

Chagrin Falls

TRIPLE K DUDE RANCH, Country Lane Road, Bainbridge

\section{Delaware County}

DUROS DUDE RANCH, INC.

Route 1, Powe11

Fairfield County

WILLOW RIDGE, William F. Ridge, Route 1, Carroll

Franklin County

BEV'S RIDING ACADEMY, 3509 Jackson Pike, Columbus

BLACKLICK RIDING \& FISHING CLUB,

1253 Waggoner, Columbus

KITSMILLER'S RIDING STABLES,

3963 Darling, Columbus
Franklin County (cont'd.)

NEDELMAN RIDING STABLES, Nathan Nedelman, Waggoner Road, Columbus

WILCOX PONY FARM, 7070 Smoky Row, Columbus

\section{Geauga County}

BROKEN BIT RANCH, Blanche Fleder, Garrettsville

RED RAIDER CAMPS, INC., Fox Smith, Russe11

ROSE FARM, Myron Rose, Russel1

$\frac{\text { Greene County }}{\text { DILLE FARM, Dr. Charles Dille, }}$
5300 Wilmington Ave., Dayton 40

Guernsey County

SMEE'S RIDING STABLE, Joe Smee, Route 6, Cambridge

\section{Hamil ton County}

HAMILTON COUNTY PARK, 1000 Miles Road, Cincinnati 31

WEST FORK RIDING ACADEMY, William R. Beckman, 2797 West Fork Road, Cincinnati 11

\section{Jefferson County}

IRVINE RIDING STABLES, John Irvine, Route 1, Richmond

KANOSKI RIDING STABLES, Herman Kanoski, Adena

MYNSTER RIDING STABLES, Patrick Mynster, Route 1, Richmond

Knox County CAMP NELSON DODD, Brinkhaven VAN RHODEN RIDING STABLE, Armor Van Rhoden, Route 5, Mt. Vernon

Lawrence County

HILLTOP FARM, William Coburn, Waterloo

OAK RIDGE HORSE FARM, William Hieronimus, Ironton

PALOMINO RANCHO, George MCClure, Route 1, Pedro 
Licking County

HITT RIDING STABLES, Vincent

Hitt, Route 1, Granville

Lorain County

BUELL'S RIDING ACADEMY, Dwight

Bue11, Route 1, Amherst

MEADOW LANE, Alton Moskal, Route 3, Wellington

MIDWAY RIDING STABLES, Lorain County

\section{Luc as County}

PEARSON PARK RIDING TRAILS, 583 S. Lallendorf, Toledo

Marion County

BLAIR RIDING STABLE, Leatha Blair, Route 1, Morral

IDLE HOUR RIDING STABLE, L. M. Wickersham, Route 2, Caledonia

\section{Medina County}

CIRCLE M STABLES, Frank Marrek, Route 1, Hinckley

MACK'S LAZY T, John C. Mack, Valley City

SLABAUGH'S RIDING ACADEMY, Joseph Slabaugh, Route 1 Medina

Meigs County

HILL FARM, Eskie Hill, Pomeroy

\section{Montgomery County}

FAR HILLS RIDING STABLES, 3563 Far Hills Avenue, Dayton 29

NORMANDY RIDING ACADEMY,

E. Raymond Arn, 1 Rossmore Court, Dayton 59

Muskingum County

STOTTS RIDING STABLES, Russel1

Stotts, Adamsville Road,

Zanesville
Noble County

TRIPLE R. RANCH, Irvil Rayner, Pleasant City

\section{Pickaway County}

EL RANCHO DARBY, George Mayer, Route 2, Orient

\section{Ross County}

CHARLES BLACK STABLES, Chillicothe LAKE HILL, Hill Brothers, Frank fort

\section{Seneca County}

TIFFIN RIDING ACADEMY, Lloyd Fruth, 112 East Market St., Tiffin

Stark County

$\mathrm{J} \& \mathrm{~J}$ RIDING ACADEMY, Jack Ames, 5704 Kemary Road, S. W., Navarre

M - BAR - K, Don Myers, McCullem Road, Alliance

\section{Summit County}

RILEY'S RIDING RANCH, Carlos Riley, 3909 Medina-Akron Road, Copley 21

DOUBLE-L RIDING ACADEMY, Louis

Ricci, Route 3, Newton Falls

Warren County
ARMCO ASSOCIATION PARK, Route 3,
Lebanon

FRISCHE'S HORSE FARM, David Frisch, Route 1, Morrow

\section{Washington County}

SIEDA, Carl L. Weppler \& Albert

Berg, Route 2, Whipple 

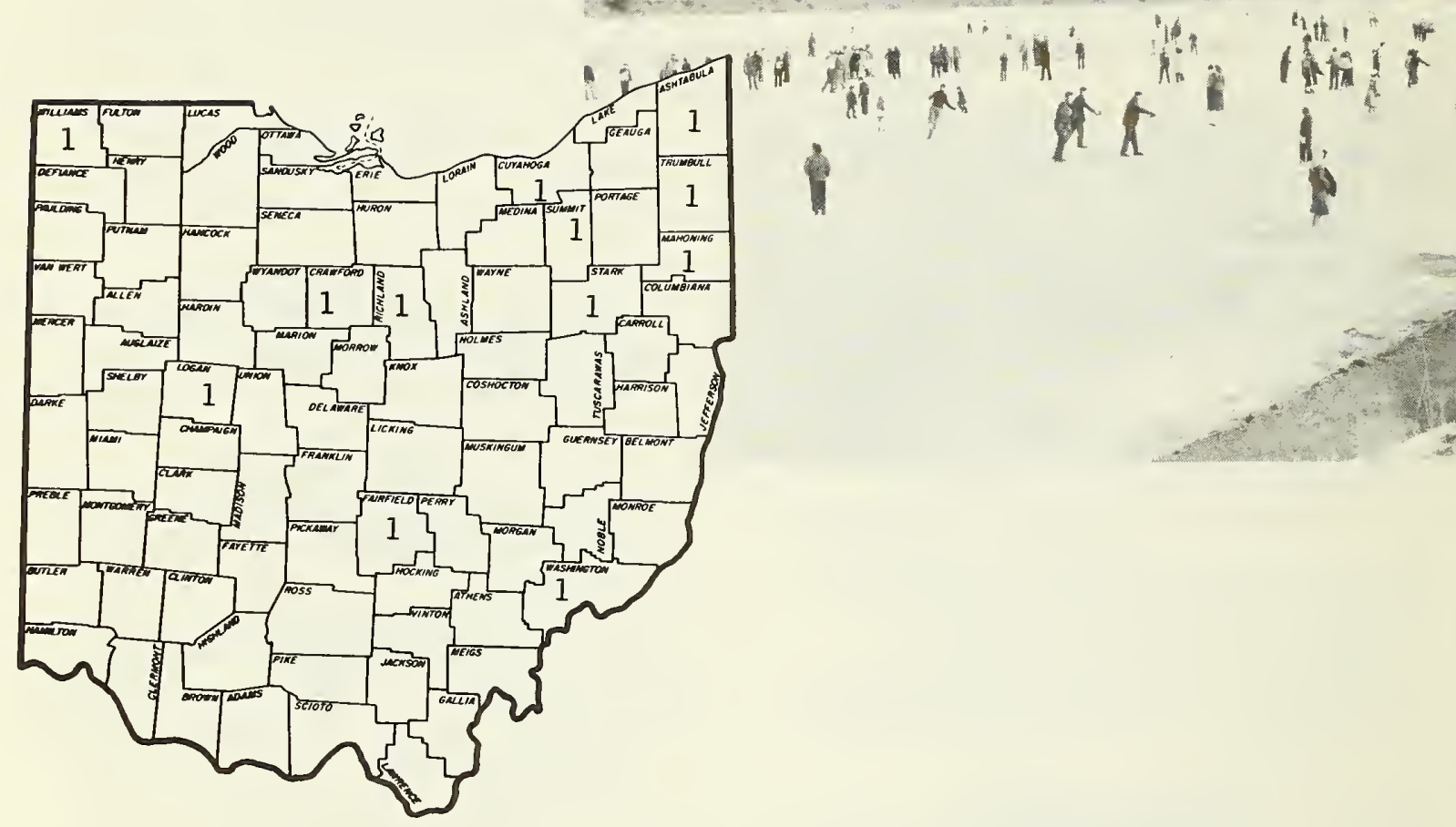

\section{WINTER SPORTS AREAS}

Winter sports areas include facilities for at least one sport such as ice skating, sledding, tobogganing, and snow skiing. Facilities are usually provided in a forest setting and often are part of other recreational enterprises, such as pay lakes, recreation parks, etc. Therefore, both facilities and services vary widely. Where the sports are tobogganing and snow skiing, facilities are often elaborate and extensive and offer every possible service to the winter sports enthusiast. Where the sports are ice skating and sledding, facilities are often primitive and simple. Besides an entrance fee, there are usually additional fees for renting equipment.

Ashtabula County

ANDOVER SKI AREA, Andover

\section{Crawford County}

SUNSET SPRINGS, George

Preston, Oceola

\section{Cuyahoga County}

SIEDEL LAKE, Norbert Siede1,

21897 Westwood Drive,

Strongsville 36

\section{Fairfield County}

ADENA RIDGE, William Burnside, Route 1, Lancaster

\section{Logan County}

VALLEY HIGH SKI SLOPE, John W. Shoots, Zanesfield

\section{Mahoning County}

WILDWOOD LAKE PARK, Carl Shank, North Lima 


\section{WINTER SPORTS (cont'd.)}

Richland County

SKI TRAILS, David Gerstner, Possum Run Rd., Mansfield

\section{Stark County}

LAKE JOSEPH, Gust Winkler, Jr., 1433 Dowing St., East Sparta

\section{Summit County}

VIRGINIA KENDALL WINTER SPORTS

AREA, Quick Road, Peninsula

Trumbul1 County

CAROMA RANCH, Mark Hal1, Route 1, Lockwood

\section{Washington County}

SIEDA, Car1 L. Weppler \& Albert Berg, Route 2, Whipple

Williams County

DREAMLAND ACRES, Edward

Brodbeck, Route 1, Montpelier 


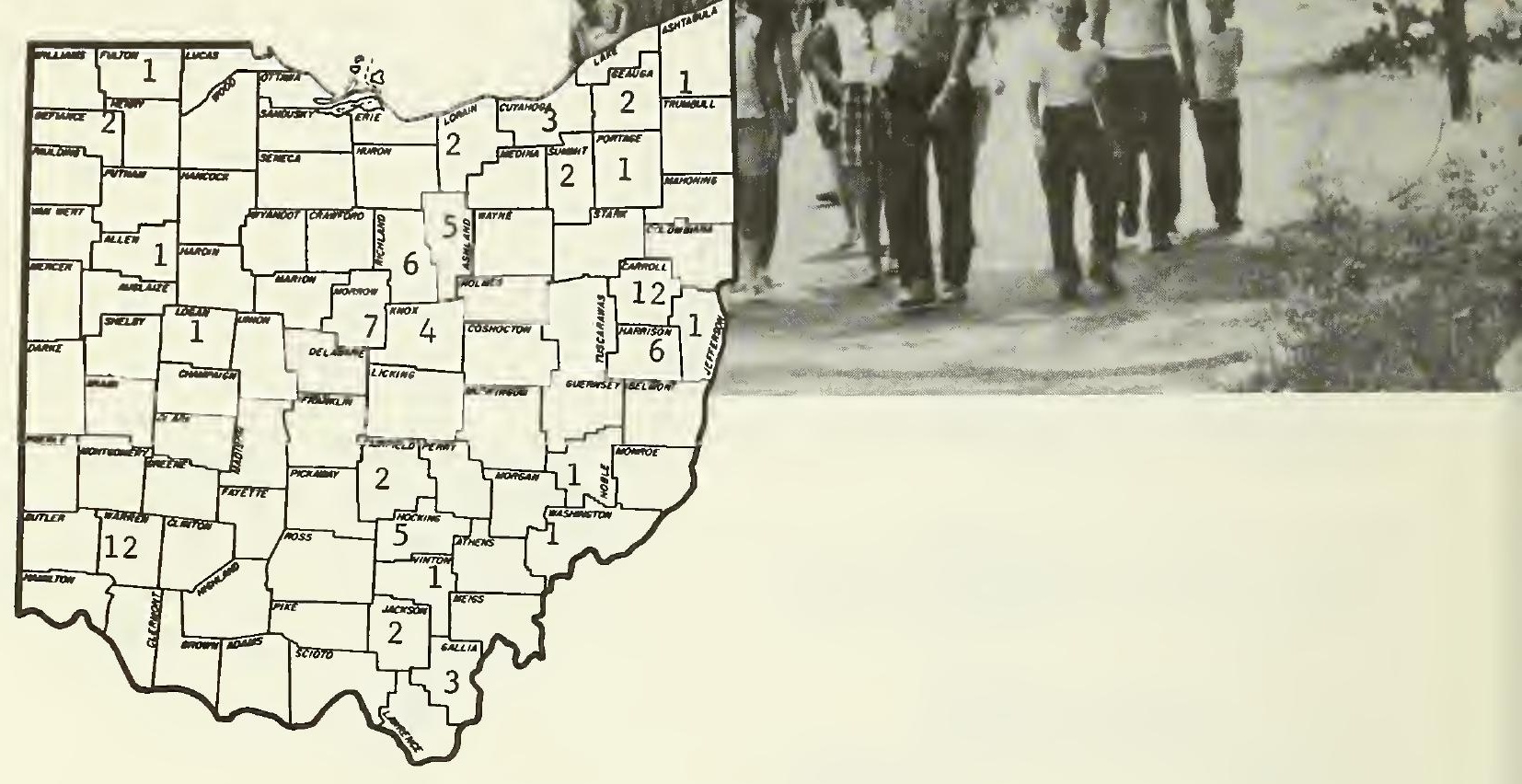

\section{ORGANIZED SUMMER CAMPS}

Summer camps serve diverse purposes, catering to particular age or interest groups such as boys or girls, the infirm, or the underprivileged. The nature and extent of facilities, as well as the camp layout, usually vary with the purpose. But the same camp may be used by several different kinds of groups and therefore is designed to satisfy a variety of needs. Whatever the purpose, camps are located in or near a natural forest environment. Living in close contact with nature is the unique feature that distinguishes camping most clearly from all other youth programs. The charge is generally on a weekly basis.

Allen County

CAMP TOKUMTO, Calvin Workman, 1906 Wende11 Ave., Lima

\section{Ashland County}

CAMP WESLEY, Rev. H. L. Borden, Route 2, Perrysville HIDDEN WOODLAND, Harold

Sponsler, Route 1, Nova
Ashland County (cont'd.) MANSFIELD GIRL SCOUT COUNCIL, Mrs. E. D. Gibson, 282 W. 4th St., Mansfield

OHIO BAPTIST CONVENTION, Bert Gilmore, Box 59, Granville ROUND LAKE CHRISTIAN ASSEMBLY, John Strader, Route 1, Lakeville 


\section{SUMMER CAMPS (cont'd.)}

Ashtabula County

LAZY ACRES, R. E. Butler, Route 2, Geneva

Carro11 County

CAMP ALDERSGATE, Rev. Charles

A. Tulga, Route 3, Carrollton

CAMP CONESTOGA, Mrs. Ester

Grossman, Route 1, Minerva

CAMP ECCO, Rev. Paul Dunn,

Route 1, Scio

CAMP FALCON, William V. Lorimer, Route 3, Carrollton

CAMP FIREBIRD, William V.

Lorimer, Route 1, Bowerstown

CAMP MUSKINGUM, Leslie Crabbe,

Route 3, Carrollton

CAMP WAKONDA, Orville Briner,

Route 1, Sherrodsville

ELKHORN VALLEY CHRISTIAN ASSOCIATION, Guy Wanless, 1005 Gilchrist St., Brilliant GREAT TRAIL GIRLS SCOUT CAMP, Mifton H. Lacey, 433 Tuscarawas St., W., Canton

ROLLING Y RANCH, Kermit Long, Route 4, Carrollton

SANDONEIDA GIRLS SCOUT CAMP, Route, Malvern

TWIN SPRUCE BOY SCOUT CAMP, John Ackert, Route 4, Carrollton

Cuyahoga County

CAMP ROBIN, Robert J. Farkas, 7843 Pearl Road, Strongsville 36

GREEN FIELDS BOYS \& GIRLS DAY CAMP, 6615 Royalton Road, Brecksville

PARKERS RANCH-HORSEMANSHIP, 23433 Mastick Road, N. Olmsted

Defiance County

CAMP LAKOTA, Route 5, Defiance

CAMP LIBBEY, Route 6, Defiance

Fairfield County

CAMP INDIANOLA, Route 2, Lancaster
Fairfield County (cont'd.)

GENEVA HILLS, Rev. Richard

Beidler, Route 4, Lancaster

Fulton County

CAMP PALMER, L. L. Spiess,

Fayette

Gallia County

CAMP ASBURG, Portsmouth

KIWANIS YOUTH CAMP, Gallipolis

WILLOW GROVE CHURCH CAMP, William

Uher, 319 E. Twelfth Ave.,

We11ston

Geauga County

CAMP REDWING, Arthur Beduhn RED RAIDER CAMPS, INC., Fox Smith, Russe11

\section{Harrison County}

AKRON BOY SCOUTS, E. H. West, Route, Tippecanoe

BOY SCOUT CAMP, Piedmont

CANTON Y.M.C.A., Perry Wolfe,

Tippecanoe

CLEVELAND BOY SCOUTS, Don Farmer, Freeport

GOSPEL TIME CHURCH CAMP, Irvin

Crosier, Route, Tippecanoe

STEUBENVILLE BOY SCOUTS, Frank

Parker, Route, Tippecanoe

\section{Hocking County}

BIG BROTHERS CAMP, Route 2, Logan

CAMP AKITA, Route 2, Logan

CAMP ALLEGRO, Robert White,

Route 2, Logan

CAMP GREEN HILL, Route 3, Logan

CAMP OTTERBEIN, Route 3, Logan

\section{Jackson County}

CAMP ARROWHEAD, Frank Longstretch and Brooks Jones, Box 388, Jackson

CANTER'S CAVE, Rodney Marhoover, Box 110, Jackson 
Jefferson County

KNIGHTS OF COLUMBUS YOUTH CAMP, Raymond Bernard, Bloomingdale

Knox County

CAMP CORNISH, Route 1,

Mt. Vernon

CAMP KOKOSING, Charles C. Ime1, Gambier

CAMP NELSON DODD, Brinkhaven

SEVENTH DAY ADVENTIST CAMP,

Route 1, Danville

Logan County

ROLLICKING HILLS, Robert

Smithers, DeGraff

Lorain County

COBB CROFT, Dr. C. O. Cobb,

Route 2, oberlin

OBERLIN DAY CAMP, Mrs. Robert

Kretchmar, Route 2, Oberlin

BROAD STREET PRESBYTERIAN

CHURCH CAMP, 760 E. Broad

St., Columbus 5

CAMP CRESWELL, Route 1, Mount Gilead

CHURCH OF GOD CAMP, Route 3 Marengo

CROOKED LANE GIRL SCOUT CAMP, Route, Galion

GODMAN GUILD ASSOCIATION CAMP, Anthony J. Salvatore, Route 2,

Fredericktown

LONE STAR CAMP, Galion

LUTHERAN MEMORIAL, Fulton

Noble County

PINE LAKE CAMP, ROY Wikander, Caldwe11

CAMP PALMER, Paige Palmer
Mantua Township
Richland County

CAMP MOWANA, Roxy Falde, Route 2, Mansfield

CAMP OTYOKWAH, Harold Smith, Route 1, Butler

TEMPLED HILLS, Morris Studenmund, Route 1, Bellville

FRIENDLY HOUSE HIDDEN HOLLOW

CAMP, Russe11 Gimbe1, Possum

Run Road, Bellville

WOODS \& WATER CHRISTIAN RETREAT,

Rev. Herbert White, Box 725,

Mansfield

WOOSTER PRESBYTERY CAMP, Neil

Eckleberry, Route 2, Perrysville

HOLIDAY HILLS DAY CAMP, 572 Hines

Hill Road, Peninsula

SHAKER DAY CAMP, 2569 old Mill

Road, Huds on

COLUMBUS $\frac{\text { Vinton County }}{\text { COMMUNITY COUNCIL, }}$

Thelma Stinson, New Plymouth

Warren County

CAMP BUTTERWORTH, Route 1, Maineville

CAMP FIREFLY, Route 1, Oregonia

CAMP GRAHAM, Clarksville

CAMP HOOK, Route 1, Oregonia

CAMP JOY, Rev. Morris McCrackin, Route 1, Clarksville

CAMP MARIANA, Route 1, Pleasant Plain

CAMP STONYBROOK, Waynesville

CAMP SWANEKY, Route 1, Oregonia

CAMP WA-KA-KEE, Route 1 , Oregonia

CAMP WHIP-OR-WILI, Jack Garrison, Route 1, Morrow

CHURCH OF GOD YOUTH CAMP, Rev. Steenbargen, Route 1 , Oregonia

KETTERING BRANCH - YMCA, Route 2, Waynesville

Washington County

CAMP HERVIDA, Lloyd Stacy, Marietta 


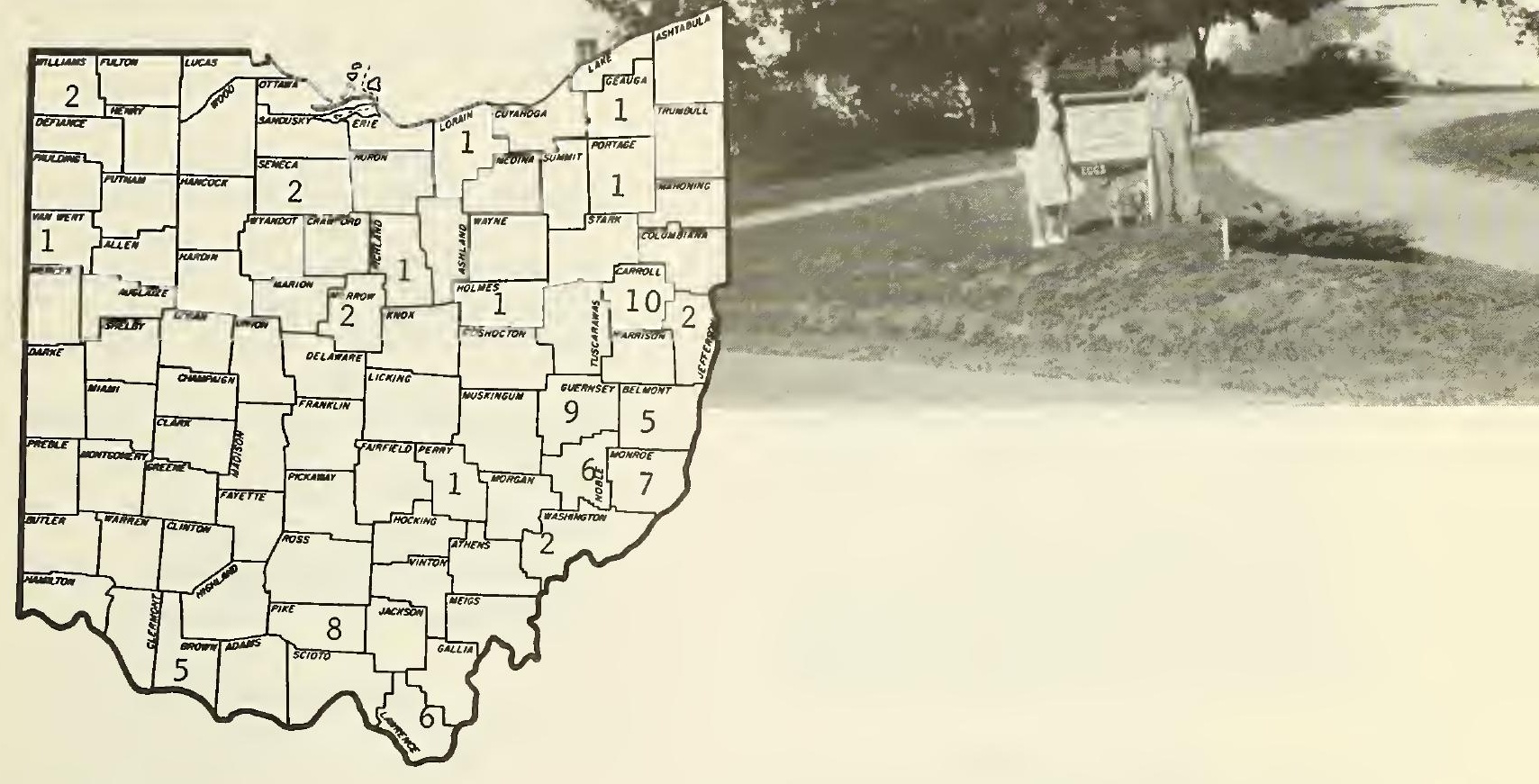

\section{VACATION FARMS AND DUDE RANCHES}

Vacation farms and dude ranches are places city dwellers go to relax and get away from urban traffic. The farm homes are usually modern with more than enough sleeping, eating, and living space. The main attractions are country air, home cooking, a woodlot, pleasant and quiet surroundings, and participation in farming activities. Other attractions may include horses to ride, a swimming hole, a place to fish, and fields and woods for hiking. The fee is usually on a daily or weekly basis.

\section{Belmont County \\ HOUSE OF SEVEN GABLES,}

A. Porterfield, Belmont

RAMBLING ACRES FARM, Sam

Ackerman, Malaga

SHEPHERD'S DAIRY FARM, Bill

Shepherd, Route 2, Belmont

SPRING VALLEY FARM, Sam Goodwin, Jerusalem

SUMMIT FARM, Charles Mitchell, Route 2, Jacobsburg

\author{
Brown County \\ BANK FARM, Leon Hesler, Route 1, \\ Winchester \\ DOWNING VACATION FARM, Lawrence \\ Downing, Route 2, Ripley \\ JONES VACATION FARM FOR BOYS, \\ Robert Jones, Route 1, \\ Georgetown \\ LAKE FRONT FARM, Floyd Fite, \\ Route 1, Bethel \\ VI-TOM-LA FARM, Thomas McNown, \\ Route 2, Georgetown
}


Carroll County

BRIAR PATCH FARM, Eugene Mitten, Dell roy

CAPPER VACATION FARM, Lawrence Capper, Route 3, Carrollton

EASTVIEW FARM, Wayne Tripp, Route 1, Bowerstown

ECHO VALLEY FARM, Franklin Fuss, Route 4, Carrollton

GARNER VACATION FARM, Bill Garner, Route 3, Carrollton

INGLEWOOD FARM, Dr. William J. Rogers, Route 4, Carrollton LOCUST GROVE FARM, Bob Newe11, Route 5, Carrollton

PINE ISLE LODGE, Lester Stearns, Sherrodsville

RINEHART VACATION FARM, DeFord Rinehart, Magnolia TWIN VALLEY FARM, Lewis Dunlap, Route 2, Carrollton

\section{Geauga County}

ROLLING GREEN FARM, John Jackson, Route 1, Burton

\section{Guernsey County}

BUSY BEE FARM, Raymond Jones, Route 1, Lore City

FOOTHILLS FARM, Frank Barnes, Route 1, Cambridge MITCHELL'S PARADISE, Walter Mitchell, Route 5, Cambridge OLD HOMESTEAD, David Blackwood, Route 3, Quaker City OUR LITTLE HOUSE, R. H. Craig, Route 1, Senecaville PERRY ORCHARD'S, Russell Perry, Route 1, Cambridge RED LANE FARM, Joe Yurina, Route 3, Cumberland SALT SPRING, Robert Hodges, Jr., Route 5, Cambridge WOODROW BROOM FARM, Woodrow Broom, Route 2, Lore City

\section{Holmes County}

TRUSSEL HOLLOW VACATION FARM, Floyd Jones, Brinkhaven
Jefferson County

SUNSET HILL FARM, John Kropp, Route 1, Richmond

YONDER HILLS FARM, John Kirk, Route 1, Bloomingdale

Lawrence County

HILLTOP FARM, William Coburn, Waterloo

OAK RIDGE HORSE FARM, William Hieronimus, Ironton

PALOMINO RANCHO, George McClure, Route 1, Pedro

POW WOW HOLLER, Bob Schweickart, Pedro

SYMMES VALLEY FARM, Frank Herre11, Route 2, Pedro TWIN HILLS, Alfred Bills, Route 2, South Point

\section{Lorain County}

R M \& R RANCH, Elyria

\section{Monroe County}

CIRCLE B FARM, Vernon E. Brague, Route 2, Clarington

HILLTOP ACRES, Snide Winland, 133 S. Main St., Woodsfield

HILLVIEW VACATION FARM, Floyd Mann, Malaga

LAKEVIEW FARM, Carl Dietrich, Route 2, Clarington

OLD-OLD FARM, Bill Dennison, Route 4, Woodsfield

SPRING VALLEY FARM, Sam Goodwin, Route 1, Jerusalem

ULLMAN HEREFORD FARM, Harry Ullman, Rinard Mills

CEDAR CREEK DUDE RANCH, Mount Gilead

ON FARM VACATION, Mrs. Harley Prosser, Route 8, Lexington

Noble County

GARRETT HEREFORD FARM, Larry Garrett, Route 1, Summerfield 
Noble County (cont'd.)

GREEN ACRES, Ralph Danford, Summerfield

LOCUST GROVE FARM, Floyd Stern, Summerfield

RIDGE HAVEN, V. Havener, Route 1, Summerfield

SCHRAMM HEREFORD FARM, Luther Schramm, Harriettsville STARR'S HILLTOP ACRES, Richard Starr, Route 1, Quaker City

\section{Perry County}

MASTERSON'S, Roy A. Masterson, Route 2, Crooksville

Pike County

BOGGS VACATION FARM, John Boggs, 304 East Fourth St., Waverly

BRUNNER VACATION FARM, Dixie Brunner, Route 2, Beaver MCALLISTER VACATION FARM, Hobart McAllister, Route 1 Rarden

MCGUIRE VACATION FARM, Mrs. S. D. McGuire, Route 1, Waverly

PICKELSIMER VACATION FARM, Martin Pickelsimer, Route 2, Beaver

SMITH VACATION FARM, Verlin Smith, Elm Grove

SMITH VACATION FARM, Tom Smith, Route 2, Piketon

WEAVER VACATION FARM, Harry Weaver, Route 3, Waverly

Portage County

SILVER SPUR, INC., LOu

Kanvasser, Ravenna-Newton

Falls Road, Ravenna

Richland County

CEDAR CREEK RANCH, Kenneth Strock, Lexington
Serec a County
MEADOWBROOK FARM, Ernest Myers, Route, Attica
PALO ALPO VACATION FARM, Stanley Cavanaugh, Route 2, Box 166, Tiffin
Van Wert County
CEMCO, C. E. McCleery, Route 1, Van Wert

Washington County

LAKESIDE, Chop Ullman, Beverly

SKYLINE FARM, Herman Ashley, Route 1, Belpre

Wi11iams County ASCHLIMAN VACATION FARM, Raymond Aschliman, Route 2, Stryker

SCHELLING VACATION FARM, Charles Schelling, Route 1 , West Unity 

